\title{
STRONG RATE OF CONVERGENCE FOR THE EULER-MARUYAMA APPROXIMATION OF STOCHASTIC DIFFERENTIAL EQUATIONS WITH IRREGULAR COEFFICIENTS
}

\author{
HOANG-LONG NGO AND DAI TAGUCHI
}

\begin{abstract}
We consider the Euler-Maruyama approximation for multi-dimensional stochastic differential equations with irregular coefficients. We provide the rate of strong convergence where the possibly discontinuous drift coefficient satisfies a one-sided Lipschitz condition and the diffusion coefficient is Hölder continuous and uniformly elliptic.
\end{abstract}

\section{INTRODUCTION}

Let us consider the $d$-dimensional stochastic differential equation (SDE)

$$
X_{t}=x_{0}+\int_{0}^{t} b\left(s, X_{s}\right) d s+\int_{0}^{t} \sigma\left(s, X_{s}\right) d W_{s}, x_{0} \in \mathbb{R}^{d}, t \in[0, T],
$$

where $W:=\left(W_{t}\right)_{0 \leq t \leq T}$ is a standard $d$-dimensional Brownian motion on a probability space $(\Omega, \mathcal{F}, \mathbb{P})$ with a filtration $\left(\mathcal{F}_{t}\right)_{0 \leq t \leq T}$ satisfying the usual conditions. The drift coefficient $b$ is a Borel-measurable function from $[0, T] \times \mathbb{R}^{d}$ into $\mathbb{R}^{d}$ and the diffusion coefficient $\sigma$ is a Borel-measurable function from $[0, T] \times \mathbb{R}^{d}$ into $\mathbb{R}^{d} \times \mathbb{R}^{d}$. In this article, we consider that elements of $\mathbb{R}^{d}$ are column vectors. The diffusion process $X:=\left(X_{t}\right)_{0 \leq t \leq T}$ is used to model many random dynamical phenomena in many fields of application, for example, mathematical finance, optimal control problem and filtering.

Since the solution of (1) is rarely analytically tractable, one often approximates $X$ by using the Euler-Maruyama scheme given by

$$
X_{t}^{(n)}=x_{0}+\int_{0}^{t} b\left(\eta_{n}(s), X_{\eta_{n}(s)}^{(n)}\right) d s+\int_{0}^{t} \sigma\left(\eta_{n}(s), X_{\eta_{n}(s)}^{(n)}\right) d W_{s}, t \in[0, T],
$$

where $\eta_{n}(s)=k T / n=: t_{k}^{(n)}$ if $s \in[k T / n,(k+1) T / n)$. It is well known that if the coefficients $b$ and $\sigma$ are Lipschitz continuous in space and 1/2-Hölder continuous in time, then the Euler-Maruyama scheme has a strong rate of convergence of $1 / 2$, (see [13]), i.e. for any $p>0$, there exists $C_{p}>0$ such that

$$
\mathbb{E}\left[\sup _{0 \leq t \leq T}\left|X_{t}-X_{t}^{(n)}\right|^{p}\right] \leq C_{p} n^{-p / 2} .
$$

Received by the editor November 10, 2013 and, in revised form, April 10, 2014, July 6, 2014, October 16, 2014, and January 24, 2015.

2010 Mathematics Subject Classification. Primary 60H35, 41A25, 60H10, 65C30.

Key words and phrases. Euler-Maruyama approximation, strong approximation, rate of convergence, stochastic differential equation, irregular coefficient.

This research was supported by grants of the Japanese government. 
The strong rate in the case of non-Lipschitz coefficients has been studied recently by using the approximation method of Yamada and Watanabe ([22, Theorem 1]) in Gyöngy and Rásonyi [8]. They have proven that for a one-dimensional SDE, if the diffusion coefficient is $(\alpha+1 / 2)$-Hölder continuous in space and the drift is the sum of a Lipschitz and a non-increasing $\gamma$-Hölder continuous function, then

$$
\mathbb{E}\left[\sup _{0 \leq t \leq T}\left|X_{t}-\tilde{X}_{t}^{(n)}\right|\right] \leq \begin{cases}C(\log n)^{-1 / 2} & \text { if } \alpha=0 \\ C\left(n^{-2 \alpha^{2}}+n^{-\alpha \gamma}\right) & \text { if } \alpha \in(0,1 / 2]\end{cases}
$$

where $\tilde{X}$ is the Euler's "polygonal" approximation of $X$ given by

$$
\tilde{X}_{t}^{(n)}=x_{0}+\int_{0}^{t} b\left(s, \tilde{X}_{\eta_{n}(s)}^{(n)}\right) d s+\int_{0}^{t} \sigma\left(s, \tilde{X}_{\eta_{n}(s)}^{(n)}\right) d W_{s}, t \in[0, T] .
$$

Yan 23] has obtained a result similar to (3) for the Euler-Maruyama scheme applied to a one-dimensional SDE with a drift which is Lipschitz continuous in space and Hölder continuous in time by using Tanaka's formula and some estimates for the local time. When the drift $b$ is not supposed to be continuous, Halidias et al. (Theorem 3.1 in 9]) have shown the convergence of the Euler-Maruyama approximation in $L^{2}$-norm (see also Theorem 2.8 in [7]). Regarding the rates of convergence, Gyöngy has shown that if $b$ satisfies the one-sided Lipschitz condition (see Definition 2.3 and $\sigma$ is locally Lipschitz, then the rate of almost sure convergence for the Euler-Maruyama polygonal approximation is of order 1/4 (see [6, Theorem 2.6]). Moreover, Bastani et al. have recently proven the strong $L^{p}$-rate $1 / 4$ for $p \geq 2$ for split-step backward Euler approximations of SDEs with discontinuous drift and Lipschitz continuous diffusion coefficients (see Theorem 5.2 in [1]).

Besides the strong approximation problem, the weak approximation for nonLipschitz coefficients SDE has also received a lot of attention. The weak rate of the Euler-Maruyama approximation when both drift and diffusion coefficients as well as payoff functions are Hölder continuous has been studied in [8, 13, 19]. KohatsuHiga et al. studied weak approximation errors for SDEs with discontinuous drift by using a perturbation method in [14. The case of locally Lipschitz coefficients has been studied extensively, too; see 10 and the references therein. It should be noted that the strong rate of approximation is very useful to implement an effective Multilevel Monte Carlo simulation scheme for approximating expectations of some functionals of $X$ (see [5]).

The goal of this article is to show that the strong rates obtained in $[8$ and $[23$. still hold even when $b$ is discontinuous. More precisely, we will investigate the strong rate of the Euler-Maruyama approximation under the assumption that the diffusion coefficient $\sigma$ is $(\alpha+1 / 2)$-Hölder continuous and the drift $b$ is one-sided Lipschitz and belongs to the class $\mathcal{A}$ of functions which is, roughly speaking, of bounded variation with respect to a Gaussian measure on $\mathbb{R}^{d}$. In particular, our result implies that the Euler-Maruyama approximation has the optimal strong rate 1/2 in the case of the Lipschitz continuous diffusion coefficient and discontinuous drift. Hence our result partly improves upon the ones in 6, 8, 23. In this article, Lemma 3.5 is the key estimation. If the drift coefficient $b$ is a Lipschitz continuous function, it is easy to prove this lemma. To obtain the same estimate with discontinuous drift, we use the result of Lemaire and Menozzi which is the Gaussian bound for the density of the Euler-Maruyama approximation (see [18, Theorem 2.1]). 
Finally, we note that SDEs with discontinuous drift appear in many applications such as optimal control and interacting infinite particle systems; see e.g. [2, 3, 16, .

Our paper is divided as follows: Section 2 introduces some notations and assumptions for our framework together with the main results. All proofs are deferred to Section 3 .

\section{MAin RESUltS}

2.1. Notations and Assumptions. We first introduce the class of functions for the drift coefficient.

Definition 2.1. Let $\mathcal{A}$ be the class of all bounded measurable functions $\zeta:[0, T] \times$ $\mathbb{R}^{d} \rightarrow \mathbb{R}$ such that there exists a sequence $\left(\zeta_{N}\right)_{N \in \mathbb{N}}$ of functions satisfying $\zeta_{N}(t, \cdot) \in$ $C^{1}\left(\mathbb{R}^{d} ; \mathbb{R}\right)$ for any $t \in[0, T]$ and the following conditions:

$\mathcal{A}(\mathrm{i})$ For any $L>0, \sup _{t \in[0, T]} \int_{|x| \leq L}\left|\zeta_{N}(t, x)-\zeta(t, x)\right| d x \rightarrow 0$ as $N \rightarrow \infty$.

$\mathcal{A}$ (ii) There exists a positive constant $K$ such that for any $x \in \mathbb{R}^{d}$,

$$
\sup _{t \in[0, T]} \sup _{N \in \mathbb{N}}\left|\zeta_{N}(t, x)\right| \leq K
$$

$\mathcal{A}$ (iii) There exists a positive constant $K$ such that for any $a \in \mathbb{R}^{d}$ and $u>0$,

$$
\sup _{t \in[0, T]} \sup _{N \in \mathbb{N}} \sum_{i=1}^{d} \int_{\mathbb{R}^{d}}\left|\partial_{i} \zeta_{N}(t, x+a)\right| \frac{e^{-\frac{|x|^{2}}{u}}}{u^{(d-1) / 2}} d x \leq K(1+\sqrt{u}),
$$

where $\partial_{i}$ is the partial derivative in space with respect to the $i$-th coordinate.

We call $\left(\zeta_{N}\right)_{N \in \mathbb{N}}$ an $\mathcal{A}$-approximation sequence of $\zeta$. This class of function $\mathcal{A}$ is similar to the one introduced in [15]. The following proposition shows that this class is quite large. Its proof is deferred to Section 3.7

Proposition 2.2. (i) If $\xi, \zeta \in \mathcal{A}$ and $\alpha, \beta \in \mathbb{R}$, then $\xi \zeta \in \mathcal{A}$ and $\alpha \xi+\beta \zeta \in \mathcal{A}$.

(ii) If $g:[0, T] \times \mathbb{R}^{d} \rightarrow \mathbb{R}$ is a bounded measurable function and $g(t, \cdot): \mathbb{R}^{d} \rightarrow \mathbb{R}$ is monotone in each variable separately, then $g \in \mathcal{A}$.

(iii) If $g$ is bounded and Lipschitz continuous in space, then $g \in \mathcal{A}$.

Using Proposition 2.2 one can easily verify that the class $\mathcal{A}$ contains the functions $\zeta(x)=|x-a| \wedge 1$ or $\zeta(x)=I_{a<x<b}$ for any $a, b \in \mathbb{R}^{d}$. On the other hand, one can verify that the function

$$
\zeta(x):= \begin{cases}\sqrt{|x|} \sin \frac{1}{x} & \text { if } x \neq 0 \\ 0 & \text { if } x=0\end{cases}
$$

is bounded and continuous on $\mathbb{R}$ but it does not belong to $\mathcal{A}$.

Definition 2.3. A function $f:[0, T] \times \mathbb{R}^{d} \rightarrow \mathbb{R}^{d}$ is called one-sided Lipschitz function in space if there exists a positive constant $K$ such that for any $(t, x, y) \in$ $[0, T] \times \mathbb{R}^{2 d}$,

$$
\langle x-y, f(t, x)-f(t, y)\rangle_{\mathbb{R}^{d}} \leq K|x-y|^{2} .
$$

Let $\mathcal{L}$ be the class of all one-sided Lipschitz functions. 
Remark 2.4. By the definition of the class $\mathcal{L}$, if $f, g \in \mathcal{L}$ and $\alpha \geq 0$, then $f+g, \alpha f \in$ $\mathcal{L}$. The one-sided Lipschitz property is closely related to the monotonicity condition introduced in [6] and the class $\mathcal{L}$ obviously contains all functions which are the sum of a Lipschitz function and a monotone decreasing $\gamma$-Hölder continuous function considered in 8]. Another example of a one-sided Lipschitz function is $f(x)=$ $(x-k) \mathbf{1}_{[k, k+1)}(x), k \in \mathbb{Z}$.

Many properties and applications of SDEs with one-sided Lipschitz drift can be found in [20].

Proposition 2.5. Let $g:[0, t] \times \mathbb{R} \rightarrow \mathbb{R}$ be a bounded and measurable function. Assume that for any $x \in \mathbb{R}, g(\cdot, x)$ is continuous; for any $t \in[0, T]$, the number of discontinuous points of $g(t,$.$) is countable and g \in \mathcal{L}$. Then $g \in \mathcal{A}$.

Proof. See, Section 3.8 .

We need the following assumptions on the coefficients $b=\left(b^{(1)}, \ldots, b^{(d)}\right)^{*}$ and $\sigma=\left(\sigma_{i, j}\right)_{1 \leq i, j \leq d}$. Here ${ }^{*}$ means transpose for the matrix.

Assumption 2.6. We assume that the coefficients $b$ and $\sigma$ are measurable functions and satisfy the following conditions:

(i) $b \in \mathcal{L}$ and $b^{(i)} \in \mathcal{A}$ for any $i=1, \ldots, d$ and there exists $K>0$ such that

$$
\sup _{(t, x) \in[0, T] \times \mathbb{R}^{d}}|b(t, x)| \leq K .
$$

(ii) $a=\sigma \sigma^{*}$ is bounded and uniformly elliptic, i.e., there exists $\lambda_{0} \geq 1$ such that for any $(t, x, \xi) \in[0, T] \times \mathbb{R}^{2 d}$,

$$
\lambda_{0}^{-1}|\xi|^{2} \leq\langle a(t, x) \xi, \xi\rangle_{\mathbb{R}^{d}} \leq \lambda_{0}|\xi|^{2} .
$$

(iii) $\sigma$ is a $(1 / 2+\alpha)$-Hölder continuous function with $\alpha \in[0,1 / 2]$ in space, i.e., there exists $K>0$ such that

$$
\sup _{(t, x, y) \in[0, T] \times \mathbb{R}^{2 d}, x \neq y} \frac{|\sigma(t, x)-\sigma(t, y)|}{|x-y|^{1 / 2+\alpha}} \leq K .
$$

Remark 2.7. Many functions satisfy Assumption 2.6(i). For example, any nonincreasing function or Lipschitz continuous function does. In particular, for $x \in \mathbb{R}$, the function $\mathbf{1}_{(-\infty, 0]}(x)-\mathbf{1}_{(0,+\infty)}(x)$ satisfies Assumption (2.6) (i). This function is the optimal drift coefficient for some stochastic control problem (see 2 or [12, p. 437]). From Proposition 2.2 and Remark 2.4, we know that if $f$ and $g$ satisfy Assumption 2.6(i) and $\alpha, \beta \geq 0$, then $\alpha f+\beta g$ also satisfies this condition. Assumption 2.6(ii) implies that the diffusion coefficient $\sigma$ is bounded i.e., for any $(t, x) \in[0, T] \times \mathbb{R}^{d},|\sigma(t, x)|=\left\{\sum_{i, j} \sigma_{i, j}^{2}(t, x)\right\}^{1 / 2} \leq \sqrt{d \lambda_{0}}$.

Assumption 2.8. The coefficients $b$ and $\sigma$ are $\beta$-Hölder continuous with $\beta \geq 1 / 2$ in time i.e., there exist $K>0$ such that for all $t, s \in[0, T]$ and $x \in \mathbb{R}^{d}$,

$$
|b(t, x)-b(s, x)|+|\sigma(t, x)-\sigma(s, x)| \leq K|t-s|^{\beta} .
$$

Remark 2.9. Veretennikov 21] has shown the following result. Assume that $b$ and $\sigma$ are bounded measurable functions such that $\sigma \sigma^{*}$ is bounded and uniformly elliptic. If $\sigma$ is $1 / 2$-Hölder continuous in $x \in \mathbb{R}$ when $d=1$ and it is Lipschitz in $x \in \mathbb{R}^{d}$ when $d \geq 2$, then there exists a unique strong solution to the stochastic differential equation (1) (see also [4,7, 16, 17, 24, for other criteria for the existence and uniqueness of the solution of SDE with non-Lipschitz coefficients). 
2.2. Main Results. We obtain the following results on the rates of the EulerMaruyama approximation in both $L^{1}$-norm and $L^{1}$-sup norm.

Theorem 2.10. Let Assumptions 2.6 and 2.8 hold. Then there exists a constant $C$ which depends on $K, T, \lambda_{0}, x_{0}, d, \alpha$ and $\beta$ such that for $d=1$,

$$
\sup _{\tau \in \mathcal{T}} \mathbb{E}\left[\left|X_{\tau}-X_{\tau}^{(n)}\right|\right] \leq \begin{cases}C(\log n)^{-1} & \text { if } \alpha=0, \\ C n^{-\alpha} & \text { if } \alpha \in(0,1 / 2]\end{cases}
$$

and for $d \geq 2$,

$$
\sup _{\tau \in \mathcal{T}} \mathbb{E}\left[\left|X_{\tau}-X_{\tau}^{(n)}\right|\right] \leq C n^{-1 / 2} \text { if } \alpha=1 / 2,
$$

where $\mathcal{T}$ is the set of all stopping times $\tau \leq T$.

Theorem 2.11. Under Assumptions 2.6 and 2.8, there exists a constant $C$ which depends on $K, T, \lambda_{0}, x_{0}, d, \alpha$ and $\beta$ such that for $d=1$,

$$
\mathbb{E}\left[\sup _{0 \leq t \leq T}\left|X_{t}-X_{t}^{(n)}\right|\right] \leq \begin{cases}C(\log n)^{-1 / 2} & \text { if } \alpha=0 \\ C n^{-2 \alpha^{2}} & \text { if } \alpha \in(0,1 / 2]\end{cases}
$$

and for $d \geq 2$,

$$
\mathbb{E}\left[\sup _{0 \leq t \leq T}\left|X_{t}-X_{t}^{(n)}\right|\right] \leq C n^{-1 / 2} \quad \text { if } \alpha=1 / 2 .
$$

The following theorem provides a bound on error in $L^{p}$-norm which is useful for designing a Multilevel Monte Carlo approximation scheme.

Theorem 2.12. Let Assumptions 2.6 and 2.8 hold. Then for any $p \geq 2$, there exists a constant $C$ which depends on $K, T, \lambda_{0}, x_{0}, d, \alpha, \beta$ and $p$ such that for $d=1$,

$$
\mathbb{E}\left[\sup _{0 \leq t \leq T}\left|X_{t}-X_{t}^{(n)}\right|^{p}\right] \leq \begin{cases}C(\log n)^{-1} & \text { if } \alpha=0, \\ C n^{-\alpha} & \text { if } \alpha \in(0,1 / 2],\end{cases}
$$

and for $d \geq 2$,

$$
\mathbb{E}\left[\sup _{0 \leq t \leq T}\left|X_{t}-X_{t}^{(n)}\right|^{p}\right] \leq C n^{-1 / 2} \quad \text { if } \alpha=1 / 2 .
$$

Remark 2.13. In Theorem 2.12, for $\alpha \in[0,1 / 2)$, the result is the same as in Gyöngy and Rásonyi [8]. But for $\alpha=1 / 2$, every moment bigger than 2 of the error is of the same order. The reason is that we deal with the discontinuous drift coefficients and the estimate of discontinuous part is of order $1 / 2$ for any $q \geq 1$ (see Lemma 3.5). The proof of Theorem 2.12 does not make use of Theorem 2.11, but only of Theorem 2.10. On the other hand, for $\alpha \in(0,1 / 2)$, using Theorem 2.12 and Jensen's inequality, we can obtain that the rate of convergence is $\alpha / 2$ in $L^{1}$-sup norm. For $\alpha \in[1 / 4,1 / 2)$, this result is better than Theorem 2.11 and for $\alpha \in(0,1 / 4]$, this result is worse than Theorem 2.11.

In many applications such as the regime switching problem, Assumption 2.8 fails to be satisfied. However, we are still able to obtain the same strong rates of convergence as above if we consider the polygonal Euler-Maruyama scheme (4) instead of the original Euler-Maruyama scheme (2).

Corollary 2.14. Assume $d=1$ and Assumption 2.6, Then all the estimates (6), (17) and (8) still hold when we replace $X_{t}^{(n)}$ with $\tilde{X}_{t}^{(n)}$. 
Corollary 2.15. Let Assumption 2.6 and 2.8 be satisfied. Then the conclusion of Theorem 2.10, 2.11 and 2.12 still hold if we replace $X_{t}^{(n)}$ with $\bar{X}_{t}^{(n)}$ defined by

$$
\bar{X}_{t}^{(n)}=x_{0}+\int_{0}^{t} b\left(s, \bar{X}_{\eta_{n}(s)}^{(n)}\right) d s+\int_{0}^{t} \sigma\left(\eta_{n}(s), \bar{X}_{\eta_{n}(s)}^{(n)}\right) d W_{s}, \quad t \in[0, T] .
$$

\section{Proof of THE MAIN THEOREMS}

Throughout this article, we will use the positive constants $C, C_{1}, C_{2}, \ldots$ and $c, c_{1}, c_{2}, \ldots$ which do not depend on $n$. Unless explicitly stated otherwise, these constants $C, C_{1}, C_{2}, \ldots$ depend only on $K, T, \lambda_{0}, x_{0}, d, \alpha$ and $\beta$; the constant $c, c_{1}, c_{2}, \ldots$ depend only on $K, \lambda_{0}, d$ and $\alpha$.

3.1. Gaussian bound for the density of the Euler-Maruyama scheme. Under Assumption 2.6(ii) and (iii), it follows from ([18, Theorem 2.1]) that the transition density $p^{(n)}\left(s, t, x, x^{\prime}\right)$ of $X_{t}^{(n)}$ between times $s$ and $t$ exists and there exist constants $C \geq 1$ and $c>0$ such that for any $x, x^{\prime} \in \mathbb{R}^{d}$ and $0 \leq j<j^{\prime} \leq n$,

$$
C^{-1} p_{c^{-1}}\left(t_{j^{\prime}}^{(n)}-t_{j}^{(n)}, x, x^{\prime}\right) \leq p^{(n)}\left(t_{j}^{(n)}, t_{j^{\prime}}^{(n)}, x, x^{\prime}\right) \leq C p_{c}\left(t_{j^{\prime}}^{(n)}-t_{j}^{(n)}, x, x^{\prime}\right),
$$

where $p_{c}\left(t-s, x, x^{\prime}\right):=\left(\frac{c}{2 \pi(t-s)}\right)^{d / 2} \exp \left(-c \frac{\left|x^{\prime}-x\right|^{2}}{2(t-s)}\right)$. Note that the constant $C$ depends on $K, \lambda_{0}, \eta, d, T$ and the constant $c$ depends on $K, \lambda_{0}, \eta, d$ but not on $T$.

The following lemma plays a crucial role in our argument.

Lemma 3.1. Assume that Assumption 2.6(ii) and (iii) hold. Then there exist $C \geq 1$ and $c>0$ such that for any $x, x^{\prime} \in \mathbb{R}^{d}, 0 \leq j<j^{\prime} \leq n$ and $t \in\left(t_{j^{\prime}-1}^{(n)}, t_{j^{\prime}}^{(n)}\right]$, we have

$$
p^{(n)}\left(t_{j}^{(n)}, t, x, x^{\prime}\right) \leq C p_{c}\left(t-t_{j}^{(n)}, x, x^{\prime}\right) .
$$

Proof. Note that for any $u \in \mathbb{R}^{d}$,

$$
\begin{aligned}
& p^{(n)}\left(t_{j^{\prime}-1}^{(n)}, t, u, x^{\prime}\right)=\left(\frac{1}{2 \pi}\right)^{d / 2} \frac{1}{\sqrt{\left(t-t_{j^{\prime}-1}^{(n)}\right)^{d} \operatorname{det} a\left(t_{j^{\prime}-1}^{(n)}, u\right)}} \times \\
& \exp \left(-\frac{\left\langle a^{-1}\left(t_{j^{\prime}-1}^{(n)}, u\right)\left(x^{\prime}-u-\left(t-t_{j^{\prime}-1}^{(n)}\right) b\left(t_{j^{\prime}-1}^{(n)}, u\right)\right),\left(x^{\prime}-u-\left(t-t_{j^{\prime}-1}^{(n)}\right) b\left(t_{j^{\prime}-1}^{(n)}, u\right)\right)\right\rangle_{\mathbb{R}^{d}}}{2\left(t-t_{j^{\prime}-1}^{(n)}\right)}\right) .
\end{aligned}
$$

Since $a^{-1}$ is uniformly elliptic, using the inequality $|x-y|^{2} \geq \frac{1}{2}|x|^{2}-|y|^{2}$ for any $x, y \in \mathbb{R}^{d}$, we obtain

$$
\begin{aligned}
& -\frac{\left\langle a^{-1}\left(t_{j^{\prime}-1}^{(n)}, u\right)\left(x^{\prime}-u-\left(t-t_{j^{\prime}-1}^{(n)}\right) b\left(t_{j^{\prime}-1}^{(n)}, u\right)\right),\left(x^{\prime}-u-\left(t-t_{j^{\prime}-1}^{(n)}\right) b\left(t_{j^{\prime}-1}^{(n)}, u\right)\right)\right\rangle_{\mathbb{R}^{d}}}{2\left(t-t_{j^{\prime}-1}^{(n)}\right)} \\
& \leq-\frac{\lambda_{0}^{-1}\left|x^{\prime}-u-\left(t-t_{j^{\prime}-1}^{(n)}\right) b\left(t_{j^{\prime}-1}^{(n)}, u\right)\right|^{2}}{2\left(t-t_{j^{\prime}-1}^{(n)}\right)} \\
& \leq-\frac{\left(2 \lambda_{0}\right)^{-1}\left|x^{\prime}-u\right|^{2}}{2\left(t-t_{j^{\prime}-1}^{(n)}\right)}+\frac{\lambda_{0}^{-1}\left(t-t_{j^{\prime}-1}^{(n)}\right)^{2}\left|b\left(t_{j^{\prime}-1}^{(n)}, u\right)\right|^{2}}{2\left(t-t_{j^{\prime}-1}^{(n)}\right)} \leq-c_{1} \frac{\left|x^{\prime}-u\right|^{2}}{2\left(t-t_{j^{\prime}-1}^{(n)}\right)}+C_{1} .
\end{aligned}
$$


Hence we have

$$
p^{(n)}\left(t_{j^{\prime}-1}^{(n)}, t, u, x^{\prime}\right) \leq C_{2} \frac{\exp \left(-c_{1} \frac{\left|x^{\prime}-u\right|^{2}}{2\left(t-t_{j^{\prime}-1}^{(n)}\right)}\right)}{\left(2 \pi\left(t-t_{j^{\prime}-1}^{(n)}\right)\right)^{d / 2}} .
$$

This estimate together with the Chapman-Kolmogorov equation and (9) yield

$$
\begin{aligned}
& p^{(n)}\left(t_{j}^{(n)}, t, x, x^{\prime}\right)=\int_{\mathbb{R}^{d}} p^{(n)}\left(t_{j}^{(n)}, t_{j^{\prime}-1}^{(n)}, x, u\right) p^{(n)}\left(t_{j^{\prime}-1}^{(n)}, t, u, x^{\prime}\right) d u \\
& \leq C_{3} \int_{\mathbb{R}^{d}} \frac{\exp \left(-c_{2} \frac{|u-x|^{2}}{2\left(t_{j^{\prime}-1}^{(n)}-t_{j}^{(n)}\right)}\right)}{\left(2 \pi\left(t_{j^{\prime}-1}^{(n)}-t_{j}^{(n)}\right)\right)^{d / 2}} \frac{\exp \left(-c_{2} \frac{\left|x^{\prime}-u\right|^{2}}{2\left(t-t_{j^{\prime}-1}^{(n)}\right)}\right)}{\left(2 \pi\left(t-t_{j^{\prime}-1}^{(n)}\right)\right)^{d / 2}} d u=C_{4} p_{c_{2}}\left(t-t_{j}^{(n)}, x, x^{\prime}\right) .
\end{aligned}
$$

We therefore obtain the desired estimate.

Corollary 3.2. Let $p_{t}^{(n)}$ be a density for $X_{t}^{(n)}$. From Lemma 3.1 with $j=0$, there exist $C \geq 1$ and $c>0$ such that for any $t \in(0, T]$ and $x \in \mathbb{R}^{d}$ we have

$$
p_{t}^{(n)}(x)=p^{(n)}\left(0, t, x_{0}, x\right) \leq C p_{c}\left(t, x_{0}, x\right) .
$$

3.2. Some auxiliary estimates. In this section, we derive a key estimation (Lemma 3.5) for proving the main theorems.

Lemma 3.3. Let $\zeta:[0, T] \times \mathbb{R}^{d} \rightarrow \mathbb{R}$ be a bounded measurable function and $\left(\zeta_{N}\right)_{N \in \mathbb{N}}$ a sequence of functions satisfying $\mathcal{A}(i)$ and $\mathcal{A}($ ii $)$ for $\zeta$. Let $\left(Y_{t}\right)_{0 \leq t \leq T}$ be a $d$ dimensional stochastic process with $Y_{0}=y_{0} \in \mathbb{R}^{d}$. Suppose that $Y_{t}$ satisfies the Gaussian bound condition on $[\kappa, T]$ for some $\kappa \in(0, T]$, i.e., there exist positive constants $C_{1}$ and $c_{1}$ such that

$$
p_{t}(y) \leq C_{1} \frac{e^{\frac{-c_{1}\left|y-y_{0}\right|^{2}}{t}}}{t^{d / 2}}, \quad t \in[\kappa, T],
$$

where $p_{t}$ is the density function of $Y_{t}$. Then

$$
\int_{\kappa}^{T} \mathbb{E}\left[\left|\zeta_{N}\left(t, Y_{t}\right)-\zeta\left(t, Y_{t}\right)\right|\right] d t \rightarrow 0, N \rightarrow \infty
$$

and if $T / n \geq \kappa$, then

$$
\int_{\frac{T}{n}}^{T} \mathbb{E}\left[\left|\zeta_{N}\left(t, Y_{\eta_{n}(t)}\right)-\zeta\left(t, Y_{\eta_{n}(t)}\right)\right|\right] d t \rightarrow 0, N \rightarrow \infty .
$$

Proof. For given $\varepsilon>0$, there exists $M \equiv M\left(\varepsilon, y_{0}, c_{1}\right)>0$ such that for any $|y| \geq M$,

$$
e^{-\frac{c_{1}\left|y-y_{0}\right|^{2}}{2 t}} \leq e^{-\frac{c_{1}\left|y-y_{0}\right|^{2}}{2 T}}<\varepsilon \text {. }
$$

From $\mathcal{A}(\mathrm{i})$, there exists $N^{\prime} \equiv N^{\prime}(\varepsilon)$ such that for any $N \geq N^{\prime}$,

$$
\int_{|y|<M}\left|\zeta_{N}(t, y)-\zeta(t, y)\right| d y<\varepsilon
$$


Therefore, for any $N \geq N^{\prime}$, using the Gaussian bound condition (11), the uniform boundedness of $\zeta_{N}$ and $\zeta$, and (14), we get

$$
\begin{aligned}
& \int_{\kappa}^{T}\left|\mathbb{E}\left[\zeta_{N}\left(t, Y_{t}\right)-\zeta\left(t, Y_{t}\right)\right]\right| d t \leq \int_{\kappa}^{T} d t \int_{\mathbb{R}^{d}} d y\left|\zeta_{N}(t, y)-\zeta(t, y)\right| p_{t}(y) \\
& \leq C_{1} \int_{\kappa}^{T} d t\left(\int_{|y|<M} d y+\int_{|y| \geq M} d y\right)\left|\zeta_{N}(t, y)-\zeta(t, y)\right| \frac{e^{\frac{-c_{1}\left|y-y_{0}\right|^{2}}{t}}}{t^{d / 2}} \\
& \leq C_{1} \int_{\kappa}^{T} \frac{1}{t^{d / 2}} d t \int_{|y|<M} d y\left|\zeta_{N}(t, y)-\zeta(t, y)\right|+C_{1} \int_{\kappa}^{T} d t \int_{|y| \geq M} d y \frac{e^{\frac{-c_{1}\left|y-y_{0}\right|^{2}}{t}}}{t^{d / 2}} \\
& \leq C_{1} T \frac{1}{\kappa^{d / 2}} \varepsilon+C_{1} T \varepsilon \int_{\mathbb{R}^{d}} \frac{e^{\frac{-c\left|y-y_{0}\right|^{2}}{2 t}}}{t^{d / 2}} d y \leq C_{T, \kappa} \varepsilon,
\end{aligned}
$$

where the constant $C_{T, \kappa}$ depends only on $T$ and $\kappa$. Hence by letting $\varepsilon$ go to 0 , we conclude (12). In the same way, we can show (13).

Corollary 3.4. Let Assumption 2.6 hold and let $\left(b_{N}^{(i)}\right)$ be an $\mathcal{A}$-approximation sequence of $b^{(i)}$ for each $i=1, \ldots, d$. Then for any $i=1, \ldots, d$ and $n \in \mathbb{N}$, we have

$$
\begin{aligned}
& \lim _{N \rightarrow \infty} \int_{\frac{T}{n}}^{T} \mathbb{E}\left[\left|b_{N}^{(i)}\left(s, X_{s}^{(n)}\right)-b_{N}^{(i)}\left(s, X_{\eta_{n}(s)}^{(n)}\right)\right|\right] d s \\
& =\int_{\frac{T}{n}}^{T} \mathbb{E}\left[\left|b^{(i)}\left(s, X_{s}^{(n)}\right)-b^{(i)}\left(s, X_{\eta_{n}(s)}^{(n)}\right)\right|\right] d s<+\infty .
\end{aligned}
$$

Proof. It follows from Lemma 3.1 that the densities of $X_{s}^{(n)}$ and $X_{\eta_{n}(s)}^{(n)}$ satisfy the Gaussian bound condition for $s \geq \frac{T}{n}$. Hence using Lemma 3.3 with $\kappa=T / n$ and the simple inequality ||$a-b|-| a^{\prime}-b^{\prime}|| \leq\left|a-a^{\prime}\right|+\left|b-b^{\prime}\right|$, we have

$$
\begin{aligned}
& \left|\int_{\frac{T}{n}}^{T} \mathbb{E}\left[\left|b_{N}^{(i)}\left(s, X_{s}^{(n)}\right)-b_{N}^{(i)}\left(s, X_{\eta_{n}(s)}^{(n)}\right)\right|\right] d s-\int_{\frac{T}{n}}^{T} \mathbb{E}\left[\left|b^{(i)}\left(s, X_{s}^{(n)}\right)-b^{(i)}\left(s, X_{\eta_{n}(s)}^{(n)}\right)\right|\right] d s\right| \\
& \leq \int_{\frac{T}{n}}^{T} \mathbb{E}\left[|| b_{N}^{(i)}\left(s, X_{s}^{(n)}\right)-b_{N}^{(i)}\left(s, X_{\eta_{n}(s)}^{(n)}\right)|-| b^{(i)}\left(s, X_{s}^{(n)}\right)-b^{(i)}\left(s, X_{\eta_{n}(s)}^{(n)}\right)||\right] d s \\
& \leq \int_{\frac{T}{n}}^{T} \mathbb{E}\left[\left|b_{N}^{(i)}\left(s, X_{s}^{(n)}\right)-b^{(i)}\left(s, X_{s}^{(n)}\right)\right|\right] d s \\
& +\int_{\frac{T}{n}}^{T} \mathbb{E}\left[\mid b_{N}^{(i)}\left(s, X_{\left.\eta_{n}(s)\right)}^{(n)}\right)-b^{(i)}\left(s, X_{\left.\eta_{n}(s)\right)}^{(n)} \mid\right] d s\right. \\
& \rightarrow 0, \text { as } N \rightarrow \infty
\end{aligned}
$$

which implies the desired result.

The above corollary is useful for proving the following key estimate.

Lemma 3.5. Let $b^{(i)} \in \mathcal{A}$ for $i=1, \ldots d$. Under Assumption 2.6 (ii) and (iii), for any $q \geq 1$, there exists $C \equiv C\left(K, T, \lambda_{0}, x_{0}, d, q\right)$ such that

$$
\sum_{i=1}^{d} \int_{0}^{T} \mathbb{E}\left[\left|b^{(i)}\left(s, X_{s}^{(n)}\right)-b^{(i)}\left(s, X_{\eta_{n}(s)}^{(n)}\right)\right|^{q}\right] d s \leq \frac{C}{\sqrt{n}} .
$$


Remark 3.6. The bound (15) is tight. Indeed, let us consider the case $d=1, x_{0}=0$, $\sigma=1$ and $b(x)=\mathbf{1}_{(-\infty, 0]}(x)-\mathbf{1}_{(0,+\infty)}(x)$. We will show that for any $q>0$, it holds that

$$
\int_{0}^{T} \mathbb{E}\left[\left|b\left(X_{s}^{(n)}\right)-b\left(X_{\eta_{n}(s)}^{(n)}\right)\right|^{q}\right] d s \geq \frac{C}{\sqrt{n}}
$$

for some constant $C>0$. Indeed, for any $s \geq T / n$, since $X_{\eta_{n}(s)}^{(n)}$ and $W_{s}-W_{\eta_{n}(s)}$ are independent,

$$
\begin{aligned}
& \mathbb{E}\left[\left|b\left(X_{s}^{(n)}\right)-b\left(X_{\eta_{n}(s)}^{(n)}\right)\right|^{q}\right]=2^{q-1} \mathbb{E}\left[\left|b\left(X_{s}^{(n)}\right)-b\left(X_{\eta_{n}(s)}^{(n)}\right)\right|\right] \\
& =2^{q} \mathbb{P}\left(\left(X_{\eta_{n}(s)}^{(n)}+\left(s-\eta_{n}(x)\right) b\left(X_{\eta_{n}(s)}^{(n)}\right)+W_{s}-W_{\eta_{n}(s)}\right) X_{\eta_{n}(s)}^{(n)} \leq 0\right) \\
& =2^{q} \int_{\mathbb{R}} d x \int_{\mathbb{R}} \mathbf{1}\left(x^{2}+\left(s-\eta_{n}(s)\right) b(x) x+y x \leq 0\right) p_{\eta_{n}(s)}^{(n)}(x) \frac{e^{-\frac{y^{2}}{2\left(s-\eta_{n}(s)\right)}}}{\sqrt{2 \pi\left(s-\eta_{n}(s)\right)}} d y \\
& \geq \int_{0}^{\infty} d x \int_{\mathbb{R}} \mathbf{1}\left(x-\left(s-\eta_{n}(s)\right)+y \leq 0\right) p_{\eta_{n}(s)}^{(n)}(x) \frac{e^{-\frac{y^{2}}{2\left(s-\eta_{n}(s)\right)}}}{\sqrt{2 \pi\left(s-\eta_{n}(s)\right)}} d y .
\end{aligned}
$$

Let $\Phi(u):=\int_{-\infty}^{u} \frac{e^{-\frac{v^{2}}{2}}}{\sqrt{2 \pi}} d v$. Then by the change of variable $z=y / \sqrt{s-\eta_{n}(s)}$, we have

$$
\mathbb{E}\left[\left|b\left(X_{s}^{(n)}\right)-b\left(X_{\eta_{n}(s)}^{(n)}\right)\right|^{q}\right] \geq \int_{0}^{\infty} p_{\eta_{n}(s)}^{(n)}(x) \Phi\left(-\frac{x-\left(s-\eta_{n}(s)\right)}{\sqrt{s-\eta_{n}(s)}}\right) d x .
$$

Recall that $x_{0}=0$. It follows from the lower bound of (9) that there exist constants $C_{1}$ and $c_{1}$ such that

$$
\begin{aligned}
& \mathbb{E}\left[\left|b\left(X_{s}^{(n)}\right)-b\left(X_{\eta_{n}(s)}^{(n)}\right)\right|^{q}\right] \geq \frac{1}{C_{1}} \int_{0}^{\infty} \frac{e^{-\frac{x^{2}}{2 c_{1} \eta_{n}(s)}}}{\sqrt{2 \pi c_{1} \eta_{n}(s)}} \Phi\left(-\frac{x-\left(s-\eta_{n}(s)\right)}{\sqrt{s-\eta_{n}(s)}}\right) d x \\
& \geq \frac{1}{C_{1}} \int_{0}^{\sqrt{s-\eta_{n}(s)}} \frac{e^{-\frac{x^{2}}{2 c_{1} \eta_{n}(s)}}}{\sqrt{2 \pi c_{1} \eta_{n}(s)}} \Phi\left(-\frac{x-\left(s-\eta_{n}(s)\right)}{\sqrt{s-\eta_{n}(s)}}\right) d x \\
& \geq \frac{1}{C_{1}} \int_{0}^{\sqrt{s-\eta_{n}(s)}} \frac{e^{-\frac{s-\eta_{n}(s)}{2 c_{1} \eta_{n}(s)}}}{\sqrt{2 \pi c_{1} \eta_{n}(s)}} \Phi\left(-\frac{\sqrt{s-\eta_{n}(s)}-\left(s-\eta_{n}(s)\right)}{\sqrt{s-\eta_{n}(s)}}\right) d x \\
& =\frac{\sqrt{s-\eta_{n}(s)}}{C_{1}} \frac{e^{-\frac{s-\eta_{n}(s)}{2 c_{1} \eta_{n}(s)}}}{\sqrt{2 \pi c_{1} \eta_{n}(s)}} \Phi\left(-\left(1-\sqrt{s-\eta_{n}(s)}\right)\right) .
\end{aligned}
$$

Moreover, using Komatsu's inequality (see [11, p. 17, Problem 1],

$$
\Phi(-|x|) \geq \frac{2 e^{-\frac{x^{2}}{2}}}{\sqrt{2 \pi}\left(|x|+\sqrt{x^{2}+4}\right)},
$$

we get for any $n \geq T$,

$$
\mathbb{E}\left[\left|b\left(X_{s}^{(n)}\right)-b\left(X_{\eta_{n}(s)}^{(n)}\right)\right|^{q}\right] \geq \frac{C_{T, c_{1}}}{\pi C_{1} \sqrt{c_{1}}} \frac{\sqrt{s-\eta_{n}(s)}}{\sqrt{\eta_{n}(s)}}, \quad \text { for any } s \geq \frac{T}{n},
$$


where the constant $C_{T, c_{1}}$ is a constant depending only on $T$ and $c_{1}$. Therefore, we have

$$
\int_{0}^{T} \mathbb{E}\left[\left|b\left(X_{s}^{(n)}\right)-b\left(X_{\eta_{n}(s)}^{(n)}\right)\right|^{q}\right] d s \geq C_{2} \int_{T / n}^{T} \frac{\sqrt{s-\eta_{n}(s)}}{\sqrt{\eta_{n}(s)}} d s \geq \frac{C_{3}}{\sqrt{n}},
$$

for $n \geq \max \{T, 2\}$. This concludes (16).

Proof of Lemma 3.5. Since $b$ is bounded, it is sufficient to prove (15) for $q=1$. Let $\left(b_{N}^{(i)}\right)$ be an $\mathcal{A}$-approximation sequence of $b^{(i)}$ for each $i=1, \ldots, d$. From Corollary 3.4, we have

$$
\begin{aligned}
& \int_{0}^{T} \mathbb{E}\left[\left|b^{(i)}\left(s, X_{s}^{(n)}\right)-b^{(i)}\left(s, X_{\eta_{n}(s)}^{(n)}\right)\right|\right] d s \\
& =\int_{0}^{\frac{T}{n}} \mathbb{E}\left[\left|b^{(i)}\left(s, X_{s}^{(n)}\right)-b^{(i)}\left(s, X_{\eta_{n}(s)}^{(n)}\right)\right|\right] d s \\
& \quad+\int_{\frac{T}{n}}^{T} \mathbb{E}\left[\left|b^{(i)}\left(s, X_{s}^{(n)}\right)-b^{(i)}\left(s, X_{\eta_{n}(s)}^{(n)}\right)\right|\right] d s \\
& \leq \frac{C_{1}}{n}+\lim _{N \rightarrow \infty} \int_{\frac{T}{n}}^{T} \mathbb{E}\left[\left|b_{N}^{(i)}\left(s, X_{s}^{(n)}\right)-b_{N}^{(i)}\left(s, X_{\eta_{n}(s)}^{(n)}\right)\right|\right] d s .
\end{aligned}
$$

So we estimate the second part of (17). Since $W_{s}-W_{\eta_{n}(s)}$ and $X_{\eta_{n}(s)}^{(n)}$ are independent, we have for $i=1, \ldots, d$,

$$
\begin{aligned}
\mathbb{E}\left[\left|b_{N}^{(i)}\left(s, X_{s}^{(n)}\right)-b_{N}^{(i)}\left(s, X_{\eta_{n}(s)}^{(n)}\right)\right|\right] & \\
= & \mathbb{E}\left[\mid b_{N}^{(i)}\left(s, X_{\eta_{n}(s)}^{(n)}+\left(s-\eta_{n}(s)\right) b\left(\eta_{n}(s), X_{\eta_{n}(s)}^{(n)}\right)\right.\right. \\
& \left.\left.\quad+\sigma\left(\eta_{n}(s), X_{\eta_{n}(s)}^{(n)}\right)\left(W_{s}-W_{\eta_{n}(s)}\right)\right)-b_{N}^{(i)}\left(s, X_{\eta_{n}(s)}^{(n)}\right) \mid\right] \\
= & \int_{\mathbb{R}^{d}} d x \int_{\mathbb{R}^{d}} d y \mid b_{N}^{(i)}\left(s, x+\left(s-\eta_{n}(s)\right) b\left(\eta_{n}(s), x\right)\right. \\
& \times p_{\eta_{n}(s)}^{(n)}(x)\left(\frac{1}{2 \pi\left(s-\eta_{n}(s)\right)}\right)^{d / 2} \exp \left(-\frac{|y|^{2}}{2\left(s-\eta_{n}(s)\right)}\right) .
\end{aligned}
$$

From the Gaussian bound condition for $p_{\eta_{n}(s)}^{(n)}$, there exists positive constants $C_{2} \geq 1$ and $c_{2}>0$ such that the last term of (18) is less than

$$
C_{2} \int_{\mathbb{R}^{d}} d x \int_{\mathbb{R}^{d}} d y\left|b_{N}^{(i)}\left(s, x+\left(s-\eta_{n}(s)\right) b\left(\eta_{n}(s), x\right)+\sigma\left(\eta_{n}(s), x\right) y\right)-b_{N}^{(i)}(s, x)\right|
$$

$$
\times\left(\frac{1}{\eta_{n}(s)}\right)^{d / 2} \exp \left(-c_{2} \frac{\left|x-x_{0}\right|^{2}}{2 \eta_{n}(s)}\right)\left(\frac{1}{s-\eta_{n}(s)}\right)^{d / 2} \exp \left(-\frac{|y|^{2}}{2\left(s-\eta_{n}(s)\right)}\right) .
$$


Applying the change of variables $z=\left(s-\eta_{n}(s)\right) b\left(\eta_{n}(s), x\right)+\sigma\left(\eta_{n}(s), x\right) y$, (19) is bounded by

$$
\begin{aligned}
& C_{2} \int_{\mathbb{R}^{d}} d x \int_{\mathbb{R}^{d}} d z \frac{\left|b_{N}^{(i)}(s, x+z)-b_{N}^{(i)}(s, x)\right|}{\left|\operatorname{det}\left(\sigma\left(\eta_{n}(s), x\right)\right)\right|}\left(\frac{1}{\eta_{n}(s)}\right)^{d / 2} \exp \left(-c_{2} \frac{\left|x-x_{0}\right|^{2}}{2 \eta_{n}(s)}\right) \\
& \times\left(\frac{1}{s-\eta_{n}(s)}\right)^{d / 2} \exp \left(-\frac{\left|\sigma^{-1}\left(\eta_{n}(s), x\right)\left(z-\left(s-\eta_{n}(s)\right) b\left(\eta_{n}(s), x\right)\right)\right|^{2}}{2\left(s-\eta_{n}(s)\right)}\right) .
\end{aligned}
$$

Since $a^{-1}$ is uniformly elliptic,

$$
\begin{aligned}
& \left|\sigma^{-1}\left(\eta_{n}(s), x\right)\left(z-\left(s-\eta_{n}(s)\right) b\left(\eta_{n}(s), x\right)\right)\right|^{2} \\
& =\left\langle a^{-1}\left(\eta_{n}(s), x\right)\left(z-\left(s-\eta_{n}(s)\right) b\left(\eta_{n}(s), x\right)\right), z-\left(s-\eta_{n}(s)\right) b\left(\eta_{n}(s), x\right)\right\rangle_{\mathbb{R}^{d}} \\
& \geq \lambda_{0}^{-1}\left|z-\left(s-\eta_{n}(s)\right) b\left(\eta_{n}(s), x\right)\right|^{2} .
\end{aligned}
$$

By the inequality $|x-y|^{2} \geq \frac{1}{2}|x|^{2}-|y|^{2}$ for any $x, y \in \mathbb{R}^{d}$, we have

$$
\begin{aligned}
& -\frac{\left|\sigma^{-1}\left(\eta_{n}(s), x\right)\left(z-\left(s-\eta_{n}(s)\right) b\left(\eta_{n}(s), x\right)\right)\right|^{2}}{2\left(s-\eta_{n}(s)\right)} \\
& \leq-\frac{\lambda_{0}^{-1}|z|^{2}}{4\left(s-\eta_{n}(s)\right)}+\frac{\lambda_{0}^{-1}\left(s-\eta_{n}(s)\right)^{2}\left|b\left(\eta_{n}(s), x\right)\right|^{2}}{2\left(s-\eta_{n}(s)\right)} \leq-c_{3} \frac{|z|^{2}}{2\left(s-\eta_{n}(s)\right)}+C_{3} .
\end{aligned}
$$

Using this estimate and Fubini's theorem, (20) is less than

$$
C_{4} \int_{\mathbb{R}^{d}} d z \int_{\mathbb{R}^{d}} d x\left|b_{N}^{(i)}(s, x+z)-b_{N}^{(i)}(s, x)\right| \frac{\exp \left(-c_{4} \frac{\left|x-x_{0}\right|^{2}}{2 \eta_{n}(s)}\right)}{\left(\eta_{n}(s)\right)^{d / 2}} \frac{\exp \left(-c_{4} \frac{|z|^{2}}{2\left(s-\eta_{n}(s)\right)}\right)}{\left(s-\eta_{n}(s)\right)^{d / 2}} .
$$

Since $b_{N}^{(i)}(s, x+z)-b_{N}^{(i)}(s, x)=\int_{0}^{1}\left\langle z, \nabla b_{N}^{(i)}(s, x+\theta z)\right\rangle_{\mathbb{R}^{d}} d \theta$, (21) is less than

$$
\begin{aligned}
C_{4} \int_{\mathbb{R}^{d}} d z \int_{\mathbb{R}^{d}} d x \int_{0}^{1} d \theta\left|\left\langle z, \nabla b_{N}^{(i)}(s, x+\theta z)\right\rangle_{\mathbb{R}^{d}}\right| \\
\times \frac{\exp \left(-c_{4} \frac{\left|x-x_{0}\right|^{2}}{2 \eta_{n}(s)}\right)}{\left(\eta_{n}(s)\right)^{d / 2}} \frac{\exp \left(-c_{4} \frac{|z|^{2}}{2\left(s-\eta_{n}(s)\right)}\right)}{\left(s-\eta_{n}(s)\right)^{d / 2}} \\
\leq C_{4} \int_{\mathbb{R}^{d}} d z \int_{\mathbb{R}^{d}} d x \int_{0}^{1} d \theta|z|\left|\nabla b_{N}^{(i)}(s, x+\theta z)\right| \\
\times \frac{\exp \left(-c_{4} \frac{\left|x-x_{0}\right|^{2}}{2 \eta_{n}(s)}\right)}{\left(\eta_{n}(s)\right)^{d / 2}} \frac{\exp \left(-c_{4} \frac{|z|^{2}}{2\left(s-\eta_{n}(s)\right)}\right)}{\left(s-\eta_{n}(s)\right)^{d / 2}} \\
\leq C_{4} \sum_{j=1}^{d} \int_{\mathbb{R}^{d}} d z \int_{\mathbb{R}^{d}} d y \int_{0}^{1} d \theta|z|\left|\partial_{j} b_{N}^{(i)}\left(s, y+x_{0}+\theta z\right)\right| \\
\times \frac{\exp \left(-c_{4} \frac{|y|^{2}}{2 \eta_{n}(s)}\right)}{\left(\eta_{n}(s)\right)^{d / 2}} \frac{\exp \left(-c_{4} \frac{|z|^{2}}{2\left(s-\eta_{n}(s)\right)}\right)}{\left(s-\eta_{n}(s)\right)^{d / 2}}
\end{aligned}
$$


where we use the change of variable $y=x-x_{0}$ in the last inequality. It follows from Fubini's theorem and condition $\mathcal{A}$ (iii) that (22) is bounded by

$$
\begin{aligned}
\frac{C_{5}}{\sqrt{\eta_{n}(s)}} & \int_{\mathbb{R}^{d}} d z \int_{0}^{1} d \theta|z| \frac{1}{\left(s-\eta_{n}(s)\right)^{d / 2}} \exp \left(-c_{4} \frac{|z|^{2}}{2\left(s-\eta_{n}(s)\right)}\right) \\
= & \frac{C_{5}}{\sqrt{\eta_{n}(s)}} \int_{\mathbb{R}^{d}} \frac{|z|}{\left(s-\eta_{n}(s)\right)^{d / 2}} \exp \left(-\frac{c_{4}}{2} \frac{|z|^{2}}{2\left(s-\eta_{n}(s)\right)}\right) \\
& \times \exp \left(-\frac{c_{4}}{2} \frac{|z|^{2}}{2\left(s-\eta_{n}(s)\right)}\right) d z .
\end{aligned}
$$

Since $|z| \exp \left(-\frac{c_{4}}{2} \frac{|z|^{2}}{2\left(s-\eta_{n}(s)\right)}\right) \leq \sqrt{\frac{2}{e c_{4}}} \sqrt{s-\eta_{n}(s)}$ for any $z \in \mathbb{R}^{d}$, (23) is less than

$$
C_{6} \sqrt{\frac{s-\eta_{n}(s)}{\eta_{n}(s)}} \leq \frac{C_{7}}{\sqrt{n \eta_{n}(s)}} .
$$

Therefore, we have

$$
\begin{aligned}
\int_{0}^{T} \mathbb{E}\left[\left|b^{(i)}\left(s, X_{s}^{(n)}\right)-b^{(i)}\left(s, X_{\eta_{n}(s)}^{(n)}\right)\right|\right] d s & \leq \frac{C_{1}}{n}+\frac{C_{7}}{\sqrt{n}} \int_{\frac{T}{n}}^{T} \frac{1}{\sqrt{\eta_{n}(s)}} d s \\
& \leq \frac{C_{1}}{n}+\frac{C_{7}}{\sqrt{n}} \int_{0}^{T} \frac{1}{\sqrt{s}} d s \leq \frac{C_{8}}{\sqrt{n}}
\end{aligned}
$$

which concludes the proof of Lemma 3.5.

3.3. Proof of Theorem 2.10, Before proving Theorem 2.10, we introduce some notation. Define

$$
Y_{t}^{(n)}=\left(Y_{t}^{(n, 1)}, \ldots, Y_{t}^{(n, d)}\right)^{*}:=X_{t}-X_{t}^{(n)}, U_{t}^{(n)}:=X_{t}^{(n)}-X_{\eta_{n}(t)}^{(n)} .
$$

Then by the definition of $X_{t}$ and $X_{t}^{(n)}$ we have

$$
\begin{aligned}
Y_{t}^{(n)}= & \int_{0}^{t}\left\{b\left(s, X_{s}\right)-b\left(\eta_{n}(s), X_{\eta_{n}(s)}^{(n)}\right)\right\} d s \\
& +\int_{0}^{t}\left\{\sigma\left(s, X_{s}\right)-\sigma\left(\eta_{n}(s), X_{\eta_{n}(s)}^{(n)}\right)\right\} d W_{s}, \\
\left\langle Y^{(n, i)}, Y^{(n, j)}\right\rangle_{t} & \\
= & \sum_{k=1}^{d} \int_{0}^{t}\left\{\sigma_{i, k}\left(s, X_{s}\right)-\sigma_{i, k}\left(\eta_{n}(s), X_{\eta_{n}(s)}^{(n)}\right)\right\} \\
& \times\left\{\sigma_{j, k}\left(s, X_{s}\right)-\sigma_{j, k}\left(\eta_{n}(s), X_{\eta_{n}(s)}^{(n)}\right)\right\} d s .
\end{aligned}
$$

The following estimation is standard (see Remark 1.2 in [8]). For the convenience of the reader we will give a proof below.

Lemma 3.7. Under Assumption 2.6)(ii) and (iii), for any $q>0$, there exist $C \equiv$ $C\left(K, T, \lambda_{0}, d, q\right)$ such that

$$
\sup _{t \in[0, T]} \mathbb{E}\left[\left|U_{t}^{(n)}\right|^{q}\right] \leq \frac{C}{n^{q / 2}} .
$$


Proof. Let $X_{t}^{(n)}=\left(X_{t}^{(n, 1)}, \ldots, X_{t}^{(n, d)}\right)^{*}$. Recall the inequality

$$
\left(\sum_{i=1}^{m} a_{i}\right)^{q} \leq m^{(q-1) \vee 0} \sum_{i=1}^{m} a_{i}^{q}
$$

for any $m \in \mathbb{N}, a_{i} \geq 0$ and $q>0$. From this and the definition of $U_{t}^{(n)}$, we have

$$
\begin{aligned}
& \left|U_{t}^{(n)}\right|^{q}=\left(\sum_{i=1}^{d}\left|X_{t}^{(n, i)}-X_{\eta_{n}(t)}^{(n, i)}\right|^{2}\right)^{q / 2} \\
& =\left(\sum_{i=1}^{d}\left|\left(t-\eta_{n}(t)\right) b^{(i)}\left(\eta_{n}(t), X_{\eta_{n}(t)}^{(n)}\right)+\sum_{j=1}^{d} \sigma_{i, j}\left(\eta_{n}(t), X_{\eta_{n}(t)}^{(n)}\right)\left(W_{t}^{j}-W_{\eta_{n}(t)}^{j}\right)\right|^{2}\right)^{q / 2} \\
& \leq C_{1}\left(\left|t-\eta_{n}(t)\right|^{q} K^{q}+\sum_{j=1}^{d} \lambda_{0}^{q / 2}\left|W_{t}^{j}-W_{\eta_{n}(t)}^{j}\right|^{q}\right)
\end{aligned}
$$

so we have

$$
\begin{aligned}
\mathbb{E}\left[\left|U_{t}^{(n)}\right|^{q}\right] & \leq C_{2}\left(\left|t-\eta_{n}(t)\right|^{q}+\sum_{j=1}^{d} \mathbb{E}\left[\left|W_{t}^{j}-W_{\eta_{n}(t)}^{j}\right|^{q}\right]\right) \\
& \leq C_{3}\left(\left|t-\eta_{n}(t)\right|^{q}+\sum_{j=1}^{d}\left|t-\eta_{n}(t)\right|^{q / 2}\right) \leq \frac{C_{4}}{n^{q / 2}} .
\end{aligned}
$$

This concludes Lemma 3.7

Proof of Theorem 2.10, Inspired by [8], we will use the approximation technique of Yamada and Watanabe (see [22, Theorem 1]). For each $\delta \in(1, \infty)$ and $\varepsilon \in(0,1)$, we can define a continuous function $\psi_{\delta, \varepsilon}: \mathbb{R} \rightarrow \mathbb{R}^{+}$with $\operatorname{supp} \psi_{\delta, \varepsilon} \subset[\varepsilon / \delta, \varepsilon]$ such that

$$
\int_{\varepsilon / \delta}^{\varepsilon} \psi_{\delta, \varepsilon}(z) d z=1 ; \quad 0 \leq \psi_{\delta, \varepsilon}(z) \leq \frac{2}{z \log \delta}, \quad z>0 .
$$

We define a function $\phi_{\delta, \varepsilon} \in C^{2}(\mathbb{R} ; \mathbb{R})$ by

$$
\phi_{\delta, \varepsilon}(x):=\int_{0}^{|x|} \int_{0}^{y} \psi_{\delta, \varepsilon}(z) d z d y .
$$

It is easy to verify that $\phi_{\delta, \varepsilon}$ has the following useful properties:

$$
\begin{aligned}
& \phi_{\delta, \varepsilon}^{\prime}(x)=\frac{x}{|x|} \phi_{\delta, \varepsilon}^{\prime}(|x|), \text { for any } x \in \mathbb{R} \backslash\{0\}, \\
& 0 \leq\left|\phi_{\delta, \varepsilon}^{\prime}(x)\right| \leq 1, \text { for any } x \in \mathbb{R} .
\end{aligned}
$$

Moreover, we define the function $\Phi_{\delta, \varepsilon}: \mathbb{R}^{d} \rightarrow \mathbb{R}$ by

$$
\Phi_{\delta, \varepsilon}(x):=\phi_{\delta, \varepsilon}(|x|)
$$


Then we also have the following useful properties:

$$
\begin{gathered}
|x| \leq \varepsilon+\Phi_{\delta, \varepsilon}(x), \text { for any } x \in \mathbb{R}^{d}, \\
\frac{\phi_{\delta, \varepsilon}^{\prime}(|x|)}{|x|} \leq \frac{\delta}{\varepsilon}, \text { for any } x \in \mathbb{R}^{d} \backslash\{0\}, \\
\phi_{\delta, \varepsilon}^{\prime \prime}( \pm|x|)=\psi_{\delta, \varepsilon}(|x|) \leq \frac{2}{|x| \log \delta} \mathbf{1}_{[\varepsilon / \delta, \varepsilon]}(|x|), \text { for any } x \in \mathbb{R}^{d} \backslash\{0\} .
\end{gathered}
$$

Note that partial differentiations of $\Phi_{\delta, \varepsilon}$ give the following: for any $x \in \mathbb{R}^{d} \backslash\{0\}$,

$$
\begin{aligned}
\partial_{i} \Phi_{\delta, \varepsilon}(x) & =\phi_{\delta, \varepsilon}^{\prime}(|x|) \frac{x_{i}}{|x|}, \\
\partial_{i}^{2} \Phi_{\delta, \varepsilon}(x) & =\phi_{\delta, \varepsilon}^{\prime \prime}(|x|) \frac{x_{i}^{2}}{|x|^{2}}+\phi_{\delta, \varepsilon}^{\prime}(|x|)\left(\frac{|x|^{2}-x_{i}^{2}}{|x|^{3}}\right), \\
\partial_{i} \partial_{j} \Phi_{\delta, \varepsilon}(x) & =\phi_{\delta, \varepsilon}^{\prime \prime}(|x|) \frac{x_{i} x_{j}}{|x|^{2}}-\phi_{\delta, \varepsilon}^{\prime}(|x|)\left(\frac{x_{i} x_{j}}{|x|^{3}}\right) .
\end{aligned}
$$

Notice also that all derivatives of $\phi_{\delta, \varepsilon}$ and $\Phi_{\delta, \varepsilon}$ at the origin are equal to 0 . In particular, note that for any $x \in \mathbb{R}^{d}$ and $i=1, \ldots, d$, using (26) and (30), we have

$$
\left|\partial_{i} \Phi_{\delta, \varepsilon}(x)\right| \leq 1
$$

Then Itô's formulas, (25) and (27) imply that

$$
\begin{aligned}
\left|Y_{t}^{(n)}\right| & \leq \varepsilon+\Phi_{\delta, \varepsilon}\left(Y_{t}^{(n)}\right) \\
& =\varepsilon+\int_{0}^{t} I_{s}^{\delta, \varepsilon, n} d s+M_{t}^{\delta, \varepsilon, n}+\frac{1}{2} \sum_{i, j=1}^{d} \int_{0}^{t} \partial_{i} \partial_{j} \Phi_{\delta, \varepsilon}\left(Y_{s}^{(n)}\right) d\left\langle Y^{(n, i)}, Y^{(n, j)}\right\rangle_{s},
\end{aligned}
$$

where

$$
I_{s}^{\delta, \varepsilon, n}:=\sum_{i=1}^{d} \partial_{i} \Phi_{\delta, \varepsilon}\left(Y_{s}^{(n)}\right)\left\{b^{(i)}\left(s, X_{s}\right)-b^{(i)}\left(\eta_{n}(s), X_{\eta_{n}(s)}^{(n)}\right)\right\}
$$

and

$$
M_{t}^{\delta, \varepsilon, n}:=\sum_{k=1}^{d} \sum_{i=1}^{d} \int_{0}^{t} \partial_{i} \Phi_{\delta, \varepsilon}\left(Y_{s}^{(n)}\right)\left\{\sigma_{i, k}\left(s, X_{s}\right)-\sigma_{i, k}\left(\eta_{n}(s), X_{\eta_{n}(s)}^{(n)}\right)\right\} d W_{s}^{k}
$$

Since $\partial_{i} \Phi_{\delta, \varepsilon}$ and $\sigma$ are bounded, $M_{t}^{\delta, \varepsilon, n}$ is a martingale. Therefore the expectation of $M_{t}^{\delta, \varepsilon, n}$ equals 0 , so we only estimate the second and fourth parts of (31). First we consider the second part. From Assumption 2.8, (25), (26) and partial 
differentiations of $\Phi_{\delta, \varepsilon}$, we have

$$
\begin{aligned}
\int_{0}^{t} I_{s}^{\delta, \varepsilon, n} d s & =\sum_{i=1}^{d} \int_{0}^{t} \phi_{\delta \varepsilon}^{\prime}\left(\left|Y_{s}^{(n)}\right|\right) \frac{Y_{s}^{(n, i)}}{\left|Y_{s}^{(n)}\right|}\left\{\left(b^{(i)}\left(s, X_{s}\right)-b^{(i)}\left(s, X_{\eta_{n}(s)}^{(n)}\right)\right)\right. \\
& \left.+\left(b^{(i)}\left(s, X_{\eta_{n}(s)}^{(n)}\right)-b^{(i)}\left(\eta_{n}(s), X_{\eta_{n}(s)}^{(n)}\right)\right)\right\} d s \\
& \leq \sum_{i=1}^{d} \int_{0}^{t} \phi_{\delta \varepsilon}^{\prime}\left(\left|Y_{s}^{(n)}\right|\right) \frac{Y_{s}^{(n, i)}}{\left|Y_{s}^{(n)}\right|}\left(b^{(i)}\left(s, X_{s}\right)-b^{(i)}\left(s, X_{\eta_{n}(s)}^{(n)}\right)\right) d s+\frac{C_{1}}{n^{\beta}} \\
& =\int_{0}^{t} \sum_{i=1}^{d} \phi_{\delta \varepsilon}^{\prime}\left(\left|Y_{s}^{(n)}\right|\right) \frac{Y_{s}^{(n, i)}}{\left|Y_{s}^{(n)}\right|}\left(b^{(i)}\left(s, X_{s}\right)-b^{(i)}\left(s, X_{s}^{(n)}\right)\right) d s \\
& +\int_{0}^{t} \sum_{i=1}^{d} \phi_{\delta \varepsilon}^{\prime}\left(\left|Y_{s}^{(n)}\right|\right) \frac{Y_{s}^{(n, i)}}{\left|Y_{s}^{(n)}\right|}\left(b^{(i)}\left(s, X_{s}^{(n)}\right)-b^{(i)}\left(s, X_{\eta_{n}(s)}^{(n)}\right)\right) d s+\frac{C_{1}}{n^{\beta}} \\
& \leq \int_{0}^{t}\left\langle X_{s}-X_{s}^{(n)}, b\left(s, X_{s}\right)-b\left(s, X_{s}^{(n)}\right)\right\rangle_{\mathbb{R}^{d}} \frac{\phi_{\delta \varepsilon}^{\prime}\left(\left|Y_{s}^{(n)}\right|\right)}{\left|Y_{s}^{(n)}\right|} d s \\
& +\sum_{i=1}^{d} \int_{0}^{t}\left|b^{(i)}\left(s, X_{s}^{(n)}\right)-b^{(i)}\left(s, X_{\eta_{n}(s)}^{(n)}\right)\right| d s+\frac{C_{1}}{n^{\beta}} .
\end{aligned}
$$

By using the one-sided Lipschitz condition (5), we have

$$
\int_{0}^{t} I_{s}^{\delta, \varepsilon, n} d s \leq K \int_{0}^{t}\left|Y_{s}^{(n)}\right| d s+\sum_{i=1}^{d} \int_{0}^{T}\left|b^{(i)}\left(s, X_{s}^{(n)}\right)-b^{(i)}\left(s, X_{\eta_{n}(s)}^{(n)}\right)\right| d s+\frac{C_{1}}{n^{\beta}} .
$$

Next we estimate the fourth part of (31). Using partial differentiations of $\Phi_{\delta, \varepsilon}$, the fourth part of (31) can be expressed as

$$
\frac{1}{2} \sum_{i, j=1}^{d} \int_{0}^{t} \partial_{i} \partial_{j} \Phi_{\delta, \varepsilon}\left(Y_{s}^{(n)}\right) d\left\langle Y^{(n, i)}, Y^{(n, j)}\right\rangle_{s}=A_{t}^{1, \delta, \varepsilon, n}+A_{t}^{2, \delta, \varepsilon, n}
$$

where

$$
A_{t}^{1, \delta, \varepsilon, n}:=\frac{1}{2} \sum_{i, j=1}^{d} \int_{0}^{t} \phi_{\delta, \varepsilon}^{\prime \prime}\left(\left|Y_{s}^{(n)}\right|\right) \frac{Y_{s}^{(n, i)} Y_{s}^{(n, j)}}{\left|Y_{s}^{(n)}\right|^{2}} d\left\langle Y^{(n, i)}, Y^{(n, j)}\right\rangle_{s}
$$

and

$$
\begin{aligned}
A_{t}^{2, \delta, \varepsilon, n} & :=\frac{1}{2} \sum_{i=1}^{d} \int_{0}^{t} \phi_{\delta, \varepsilon}^{\prime}\left(\left|Y_{s}^{(n)}\right|\right)\left(\frac{\left|Y_{s}^{(n)}\right|^{2}-\left|Y_{s}^{(n, i)}\right|^{2}}{\left|Y_{s}^{(n)}\right|^{3}}\right) d\left\langle Y^{(n, i)}, Y^{(n, i)}\right\rangle_{s} \\
& +\sum_{1 \leq i<j \leq d} \int_{0}^{t}\left\{-\phi_{\delta, \varepsilon}^{\prime}\left(\left|Y_{s}^{(n)}\right|\right) \frac{Y_{s}^{(n, i)} Y_{s}^{(n, j)}}{\left|Y_{s}^{(n)}\right|^{3}}\right\} d\left\langle Y^{(n, i)}, Y^{(n, j)}\right\rangle_{s} .
\end{aligned}
$$


Here we remark that $A_{t}^{2, \delta, \varepsilon, n}=0$ for $d=1$. So we should estimate $A_{t}^{1, \delta, \varepsilon, n}$ and $A_{t}^{2, \delta, \varepsilon, n}$. By the definition of quadratic variation of $Y_{t}^{(n)}$,

$$
\begin{aligned}
A_{t}^{1, \delta, \varepsilon, n} & \leq \frac{1}{2} \sum_{k=1}^{d} \sum_{i, j=1}^{d} \int_{0}^{t} \phi_{\delta, \varepsilon}^{\prime \prime}\left(\left|Y_{s}^{(n)}\right|\right) \frac{\left|Y_{s}^{(n, i)}\right|\left|Y_{s}^{(n, j)}\right|}{\left|Y_{s}^{(n)}\right|^{2}}\left|\sigma_{i, k}\left(s, X_{s}\right)-\sigma_{i, k}\left(\eta_{n}(s), X_{\eta_{n}(s)}^{(n)}\right)\right| \\
& \times\left|\sigma_{j, k}\left(s, X_{s}\right)-\sigma_{j, k}\left(\eta_{n}(s), X_{\eta_{n}(s)}^{(n)}\right)\right| d s
\end{aligned}
$$

and

$$
\begin{aligned}
A_{t}^{2, \delta, \varepsilon, n} \leq & \frac{1}{2} \sum_{k, i=1}^{d} \int_{0}^{t} \phi_{\delta, \varepsilon}^{\prime}\left(\left|Y_{s}^{(n)}\right|\right)\left(\frac{\left|Y_{s}^{(n)}\right|^{2}-\left|Y_{s}^{(n, i)}\right|^{2}}{\left|Y_{s}^{(n)}\right|^{3}}\right) \\
& \times\left|\sigma_{i, k}\left(s, X_{s}\right)-\sigma_{i, k}\left(\eta_{n}(s), X_{\eta_{n}(s)}^{(n)}\right)\right|^{2} d s \\
& +\sum_{k=1}^{d} \sum_{1 \leq i<j \leq d} \int_{0}^{t} \phi_{\delta, \varepsilon}^{\prime}\left(\left|Y_{s}^{(n)}\right|\right) \frac{\left|Y_{s}^{(n, i)}\right|\left|Y_{s}^{(n, j)}\right|}{\left|Y_{s}^{(n)}\right|^{3}} \\
& \times\left|\sigma_{i, k}\left(s, X_{s}\right)-\sigma_{i, k}\left(\eta_{n}(s), X_{\eta_{n}(s)}^{(n)}\right)\right| \\
& \times\left|\sigma_{j, k}\left(s, X_{s}\right)-\sigma_{j, k}\left(\eta_{n}(s), X_{\eta_{n}(s)}^{(n)}\right)\right| d s .
\end{aligned}
$$

Since $\sigma$ is $(1 / 2+\alpha)$-Hölder continuous in space and $\beta$-Hölder continuous in time, we have

$$
\begin{aligned}
A_{t}^{1, \delta, \varepsilon, n} & \leq C_{2} \sum_{i, j=1}^{d} \int_{0}^{t} \phi_{\delta, \varepsilon}^{\prime \prime}\left(\left|Y_{s}^{(n)}\right|\right) \frac{\left|Y_{s}^{(n, i)}\right|\left|Y_{s}^{(n, j)}\right|}{\left|Y_{s}^{(n)}\right|^{2}}\left\{\left|X_{s}-X_{\eta_{n}(s)}^{(n)}\right|^{1+2 \alpha}+\frac{1}{n^{2 \beta}}\right\} d s \\
& \leq C_{3} \int_{0}^{t} \phi_{\delta, \varepsilon}^{\prime \prime}\left(\left|Y_{s}^{(n)}\right|\right)\left\{\left|X_{s}-X_{\eta_{n}(s)}^{(n)}\right|^{1+2 \alpha}+\frac{1}{n^{2 \beta}}\right\} d s \\
& \leq C_{4} \int_{0}^{t} \phi_{\delta, \varepsilon}^{\prime \prime}\left(\left|Y_{s}^{(n)}\right|\right)\left\{\left|Y_{s}^{(n)}\right|^{1+2 \alpha}+\left|U_{s}^{(n)}\right|^{1+2 \alpha}+\frac{1}{n^{2 \beta}}\right\} d s .
\end{aligned}
$$

Similarly, we obtain

$$
A_{t}^{2, \delta, \varepsilon, n} \leq C_{5} \int_{0}^{t} \frac{\phi_{\delta, \varepsilon}^{\prime}\left(\left|Y_{s}^{(n)}\right|\right)}{\left|Y_{s}^{(n)}\right|}\left\{\left|Y_{s}^{(n)}\right|^{1+2 \alpha}+\left|U_{s}^{(n)}\right|^{1+2 \alpha}+\frac{1}{n^{2 \beta}}\right\} d s
$$

It follows from (29) that

$$
\begin{aligned}
A_{t}^{1, \delta, \varepsilon, n} & \leq C_{6} \int_{0}^{t} \frac{\mathbf{1}_{[\varepsilon / \delta, \varepsilon]}\left(\left|Y_{s}^{(n)}\right|\right)}{\left|Y_{s}^{(n)}\right| \log \delta}\left\{\left|Y_{s}^{(n)}\right|^{1+2 \alpha}+\left|U_{s}^{(n)}\right|^{1+2 \alpha}+\frac{1}{n^{2 \beta}}\right\} d s \\
& \leq \frac{C_{6} \varepsilon^{2 \alpha}}{\log \delta}+\frac{C_{6} \delta}{\varepsilon \log \delta} \int_{0}^{T}\left|U_{s}^{(n)}\right|^{1+2 \alpha} d s+\frac{C_{6} \delta}{\varepsilon(\log \delta) n^{2 \beta}},
\end{aligned}
$$


and from (26) and (28) that

$$
A_{t}^{2, \delta, \varepsilon, n} \leq C_{5} \int_{0}^{t}\left|Y_{s}^{(n)}\right|^{2 \alpha} d s+\frac{C_{5} \delta}{\varepsilon} \int_{0}^{T}\left|U_{s}^{(n)}\right|^{1+2 \alpha} d s+\frac{C_{5} \delta}{\varepsilon n^{2 \beta}} .
$$

Let $\tau$ be a stopping time with $\tau \leq T$. Define $Z_{t}^{(n)}:=\left|Y_{t \wedge \tau}^{(n)}\right|$ and for any $\alpha \in[0,1 / 2]$,

$$
R(\alpha, \delta, \varepsilon, n):=\varepsilon+\frac{C_{6} \varepsilon^{2 \alpha}}{\log \delta}+\frac{C_{6} \delta}{\varepsilon \log \delta} \int_{0}^{T}\left|U_{s}^{(n)}\right|^{1+2 \alpha} d s+\frac{C_{6} \delta}{\varepsilon(\log \delta) n^{2 \beta}}
$$

and

$$
S(\alpha, \delta, \varepsilon, n):=\frac{C_{5} \delta}{\varepsilon} \int_{0}^{T}\left|U_{s}^{(n)}\right|^{1+2 \alpha} d s+\frac{C_{5} \delta}{\varepsilon n^{2 \beta}} .
$$

Then we consider the following two cases.

Case $1(d \geq 2$ and $\alpha=1 / 2)$ : In this case, gathering the above estimates, we have

$$
\begin{array}{r}
Z_{t}^{(n)} \leq C_{7} \int_{0}^{t} Z_{s}^{(n)} d s+\sum_{i=1}^{d} \int_{0}^{T}\left|b^{(i)}\left(s, X_{s}^{(n)}\right)-b^{(i)}\left(s, X_{\eta_{n}(s)}^{(n)}\right)\right| d s \\
+\frac{C_{7}}{n^{\beta}}+M_{t \wedge \tau}^{\delta, \varepsilon, n}+R(1 / 2, \delta, \varepsilon, n)+S(1 / 2, \delta, \varepsilon, n) .
\end{array}
$$

We choose $\delta=2$ and $\varepsilon=n^{-1 / 2}$. Then for any $\alpha \in(0,1 / 2]$, we obtain

$$
R\left(\alpha, 2, n^{-1 / 2}, n\right) \leq \frac{C_{8}}{n^{\alpha}}+C_{8} \sqrt{n} \int_{0}^{T}\left|U_{s}^{(n)}\right|^{1+2 \alpha} d s+\frac{C_{8}}{n^{2 \beta-1 / 2}}
$$

and

$$
S\left(\alpha, 2, n^{-1 / 2}, n\right) \leq C_{9} \sqrt{n} \int_{0}^{T}\left|U_{s}^{(n)}\right|^{1+2 \alpha} d s+\frac{C_{9}}{n^{2 \beta-1 / 2}} .
$$

Note that $2 \beta-1 / 2 \geq 1 / 2$. It follows from Lemma 3.7 with $q=1+2 \alpha$ that for any $\alpha \in(0,1 / 2]$,

$$
\mathbb{E}\left[R\left(\alpha, 2, n^{-1 / 2}, n\right)\right], \mathbb{E}\left[S\left(\alpha, 2, n^{-1 / 2}, n\right)\right] \leq \frac{C_{10}}{n^{\alpha}} .
$$

Recall $\alpha=1 / 2$. By using the above estimate and Lemma 3.5, we obtain

$$
\mathbb{E}\left[Z_{t}^{(n)}\right] \leq C_{7} \int_{0}^{t} \mathbb{E}\left[Z_{s}^{(n)}\right] d s+\frac{C_{11}}{\sqrt{n}}
$$

By Gronwall's inequality, we have

$$
\mathbb{E}\left[Z_{t}^{(n)}\right] \leq \frac{C_{12}}{\sqrt{n}}
$$

Therefore, from the dominated convergence theorem, we complete the statement taking $t \rightarrow T$. 
Case $2(d=1)$ : As remarked before, $A_{t}^{2, \delta, \varepsilon, n}=0$. From (33) and (34), we have

$$
\begin{aligned}
Z_{t}^{(n)} \leq & C_{6} \int_{0}^{t} Z_{s}^{(n)} d s+\int_{0}^{T}\left|b\left(s, X_{s}^{(n)}\right)-b\left(s, X_{\eta_{n}(s)}^{(n)}\right)\right| d s \\
& +\frac{C_{6}}{n^{\beta}}+M_{t \wedge \tau}^{\delta, \varepsilon, n}+R(\alpha, \delta, \varepsilon, n) .
\end{aligned}
$$

For $\alpha \in(0,1 / 2]$, we can prove the statement in (6) in the same way as Case 1 by taking $\delta=2$ and $\varepsilon=n^{-1 / 2}$. For $\alpha=0$, we choose $\delta=n^{1 / 3}$ and $\varepsilon=(\log n)^{-1}$. Then we have

$$
R\left(0, n^{1 / 3},(\log n)^{-1}, n\right) \leq \frac{C_{13}}{\log n}+C_{13} n^{1 / 3} \int_{0}^{T}\left|U_{s}^{(n)}\right| d s+\frac{C_{13}}{n^{2 \beta-1 / 3}}
$$

and so we get

$$
\mathbb{E}\left[R\left(0, n^{1 / 3},(\log n)^{-1}, n\right)\right] \leq \frac{C_{14}}{\log n}
$$

From Lemma 3.5, 3.7 and (38), we have

$$
\mathbb{E}\left[Z_{t}^{(n)}\right] \leq C_{6} \int_{0}^{t} \mathbb{E}\left[Z_{s}^{(n)}\right] d s+\frac{C_{15}}{\log n}
$$

Hence by Gronwall's inequality we see that

$$
\mathbb{E}\left[Z_{t}^{(n)}\right] \leq \frac{C_{16}}{\log n}
$$

Therefore, from the dominated convergence theorem, we obtain (6) for $\alpha=0$ by taking $t \rightarrow T$.

3.4. Proof of Theorem 2.11. Recalling (24), we define $V_{t}^{(n)}:=\sup _{0 \leq s \leq t}\left|Y_{s}^{(n)}\right|$. To estimate the expectation of $V_{t}^{(n)}$, we use (35) and therefore we need to calculate the expectation of $\sup _{0 \leq s \leq t}\left|M_{s}^{\delta, \varepsilon, n}\right|$. We use the notation $\widetilde{C}_{i}$ for a positive constant instead of $C_{i}$. This constant $\widetilde{C}_{i}$ can depend on $K, T, \alpha$ and $\beta$ while the constant $C_{i}$ can be depend on $K, T, \lambda_{0}, x_{0}, \beta$ and $d$. For any $d \in \mathbb{N}$, by using (32) and the Burkholder-Davis-Gundy inequality we have

$$
\begin{aligned}
& \mathbb{E}\left[\sup _{0 \leq s \leq t}\left|M_{s}^{\delta, \varepsilon, n}\right|\right] \leq \widetilde{C}_{1} \mathbb{E}\left[\left\langle M^{\delta, \varepsilon, n}\right\rangle_{t}^{1 / 2}\right] \\
& =\widetilde{C}_{1} \mathbb{E}\left[\left(\sum_{k=1}^{d} \int_{0}^{t}\left|\sum_{i=1}^{d} \partial_{i} \Phi_{\delta, \varepsilon}\left(Y_{s}^{(n)}\right)\left\{\sigma_{i, k}\left(s, X_{s}\right)-\sigma_{i, k}\left(\eta_{n}(s), X_{\eta_{n}(s)}^{(n)}\right)\right\}\right|^{2} d s\right)^{1 / 2}\right] .
\end{aligned}
$$


Since $\partial_{i} \Phi_{\delta, \varepsilon},(i=1, \ldots, d)$ are bounded and $\sigma$ is $1 / 2+\alpha$-Hölder continuous in space and $\beta$-Hölder continuous in time, we have

$$
\begin{aligned}
& \mathbb{E}\left[\sup _{0 \leq s \leq t}\left|M_{s}^{\delta, \varepsilon, n}\right|\right] \\
& \leq \widetilde{C}_{2} \mathbb{E}\left[\left(\sum _ { i , k = 1 } ^ { d } \int _ { 0 } ^ { t } \left\{\left|\sigma_{i, k}\left(s, X_{s}\right)-\sigma_{i, k}\left(s, X_{s}^{(n)}\right)\right|^{2}\right.\right.\right. \\
& +\left|\sigma_{i, k}\left(s, X_{s}^{(n)}\right)-\sigma_{i, k}\left(s, X_{\eta_{n}(s)}^{(n)}\right)\right|^{2} \\
& \left.\left.+\mid \sigma_{i, k}\left(s, X_{\eta_{n}(s)}^{(n)}\right)-\sigma_{i, k}\left(\eta_{n}(s),\left.X_{\eta_{n}(s)}^{(n)}\right|^{2}\right\} d s\right)^{1 / 2}\right] \\
& =\widetilde{C}_{2} \mathbb{E}\left[\left(\int _ { 0 } ^ { t } \left\{\left|\sigma\left(s, X_{s}\right)-\sigma\left(s, X_{s}^{(n)}\right)\right|^{2}+\left|\sigma\left(s, X_{s}^{(n)}\right)-\sigma\left(s, X_{\eta_{n}(s)}^{(n)}\right)\right|^{2}\right.\right.\right. \\
& \left.\left.\left.+\left|\sigma\left(s, X_{\eta_{n}(s)}^{(n)}\right)-\sigma\left(\eta_{n}(s), X_{\eta_{n}(s)}^{(n)}\right)\right|^{2}\right\} d s\right)^{1 / 2}\right] \\
& \leq \widetilde{C}_{3} \mathbb{E}\left[\left(\int _ { 0 } ^ { t } \left\{\left|X_{s}-X_{s}^{(n)}\right|^{1+2 \alpha}+\left|X_{s}^{(n)}-X_{\eta_{n}(s)}^{(n)}\right|^{1+2 \alpha}\right.\right.\right. \\
& \left.\left.\left.\quad+\left|s-\eta_{n}(s)\right|^{2 \beta}\right\} d s\right)^{1 / 2}\right] \\
& \leq \widetilde{C}_{4}\left\{\mathbb{E}\left[A_{t}^{(n)}+B_{t}^{(n)}\right]+\frac{1}{\left.n^{\beta}\right\}},\right.
\end{aligned}
$$

where by the definition of $Y^{(n)}$ and $U^{(n)}$ given in (24),

$$
A_{t}^{(n)}:=\left(\int_{0}^{t}\left|Y_{s}^{(n)}\right|^{1+2 \alpha} d s\right)^{1 / 2} \quad \text { and } \quad B_{t}^{(n)}:=\left(\int_{0}^{t}\left|U_{s}^{(n)}\right|^{1+2 \alpha} d s\right)^{1 / 2} .
$$

From Lemma 3.7 with $q=1+2 \alpha$ and using Jensen's inequality, we have

$$
\mathbb{E}\left[B_{t}^{(n)}\right] \leq\left(\int_{0}^{T} \mathbb{E}\left[\left|U_{s}^{(n)}\right|^{1+2 \alpha}\right] d s\right)^{1 / 2} \leq \frac{C_{1}}{n^{1 / 4+\alpha / 2}}
$$

Next we estimate $A_{t}^{(n)}$ and $V_{t}^{(n)}=\sup _{0 \leq s \leq t}\left|Y_{s}^{(n)}\right|$ for the following two cases.

Case $1(d \geq 2$ and $\alpha=1 / 2)$ : Since $\left|Y_{s}^{(n)}\right| \leq V_{t}^{(n)}$ for all $s \leq t$, we have

$$
\mathbb{E}\left[A_{t}^{(n)}\right]=\mathbb{E}\left[\left(\int_{0}^{t}\left|Y_{s}^{(n)}\right|^{1+2 \alpha} d s\right)^{1 / 2}\right] \leq \mathbb{E}\left[\left(V_{t}^{(n)}\right)^{1 / 2}\left(\int_{0}^{t}\left|Y_{s}^{(n)}\right|^{2 \alpha} d s\right)^{1 / 2}\right] .
$$

Using Young's inequality $x y \leq \frac{x^{2}}{2 \widetilde{C}_{4}}+\frac{\widetilde{C}_{4} y^{2}}{2}$, for any $x, y \geq 0$ and Theorem 2.10, as $\alpha=1 / 2$, we get

$$
\mathbb{E}\left[A_{t}^{(n)}\right] \leq \frac{1}{2 \widetilde{C}_{4}} \mathbb{E}\left[V_{t}^{(n)}\right]+\frac{\widetilde{C}_{4}}{2} \int_{0}^{T} \mathbb{E}\left[\left|Y_{s}^{(n)}\right|\right] d s \leq \frac{1}{2 \widetilde{C}_{4}} \mathbb{E}\left[V_{t}^{(n)}\right]+\frac{C_{2}}{\sqrt{n}}
$$


Therefore as $\beta \geq 1 / 2$, we have, using (39), (40) and (41),

$$
\mathbb{E}\left[\sup _{0 \leq s \leq t}\left|M_{s}^{\delta, \varepsilon, n}\right|\right] \leq \frac{1}{2} \mathbb{E}\left[V_{t}^{(n)}\right]+C_{3}\left\{\frac{1}{\sqrt{n}}+\frac{1}{n^{\beta}}\right\} \leq \frac{1}{2} \mathbb{E}\left[V_{t}^{(n)}\right]+\frac{C_{4}}{\sqrt{n}} .
$$

Taking supremum in (35) with $\tau=T$, we obtain

$$
\begin{aligned}
V_{t}^{(n)} \leq C_{5} \int_{0}^{t} V_{s}^{(n)} d s & +\sum_{i=1}^{d} \int_{0}^{T}\left|b^{(i)}\left(s, X_{s}^{(n)}\right)-b^{(i)}\left(s, X_{\eta_{n}(s)}^{(n)}\right)\right| d s \\
& +\frac{C_{5}}{n^{\beta}}+\sup _{0 \leq s \leq t}\left|M_{s}^{\delta, \varepsilon, n}\right|+R(1 / 2, \delta, \varepsilon, n)+S(1 / 2, \delta, \varepsilon, n) .
\end{aligned}
$$

From (36), (42) and (43), we have

$$
\mathbb{E}\left[V_{t}^{(n)}\right] \leq C_{6} \int_{0}^{t} \mathbb{E}\left[V_{s}^{(n)}\right] d s+\frac{C_{6}}{\sqrt{n}} .
$$

From Gronwall's inequality we have

$$
\mathbb{E}\left[V_{t}^{(n)}\right] \leq \frac{C_{7}}{\sqrt{n}} .
$$

Case $2(d=1)$ : For $\alpha \in(0,1 / 2]$, by using the same method as in Case 1 , we have that (41) becomes

$$
\mathbb{E}\left[A_{t}^{(n)}\right] \leq \frac{1}{2 \widetilde{C}_{4}} \mathbb{E}\left[V_{t}^{(n)}\right]+\frac{\widetilde{C}_{4}}{2} \int_{0}^{T}\left(\mathbb{E}\left[\left|Y_{s}^{(n)}\right|\right]\right)^{2 \alpha} d s \leq \frac{1}{2 \widetilde{C}_{4}} \mathbb{E}\left[V_{t}^{(n)}\right]+\frac{C_{8}}{n^{2 \alpha^{2}}} .
$$

Therefore from (39), using (40) and (44) we obtain

$$
\begin{aligned}
\mathbb{E}\left[\sup _{0 \leq s \leq t}\left|M_{s}^{\delta, \varepsilon, n}\right|\right] & \leq \frac{1}{2} \mathbb{E}\left[V_{t}^{(n)}\right]+C_{9}\left\{\frac{1}{n^{2 \alpha^{2}}}+\frac{1}{n^{1 / 4+\alpha / 2}}+\frac{1}{n^{\beta}}\right\} \\
& \leq \frac{1}{2} \mathbb{E}\left[V_{t}^{(n)}\right]+\frac{C_{10}}{n^{2 \alpha^{2}}} .
\end{aligned}
$$

Taking supremum in (37) with $\tau=T$, we have

$$
\begin{aligned}
V_{t}^{(n)} & \leq C_{11} \int_{0}^{t} V_{s}^{(n)} d s+\int_{0}^{T}\left|b\left(s, X_{s}^{(n)}\right)-b\left(s, X_{\eta_{n}(s)}^{(n)}\right)\right| d s \\
& +\frac{C_{11}}{n^{\beta}}+\sup _{0 \leq s \leq t}\left|M_{s}^{\delta, \varepsilon, n}\right|+R(\alpha, \delta, \varepsilon, n) .
\end{aligned}
$$

Therefore by using (36), (45) and applying Gronwall's inequality we have

$$
\mathbb{E}\left[V_{t}^{(n)}\right] \leq \frac{C_{12}}{n^{2 \alpha^{2}}}
$$

For $\alpha=0$, it follows from Theorem 2.10 that we have

$$
\mathbb{E}\left[A_{t}^{(n)}\right] \leq\left(\int_{0}^{T} \mathbb{E}\left[\left|Y_{s}^{(n)}\right|\right] d s\right)^{1 / 2} \leq \frac{C_{13}}{\sqrt{\log n}} .
$$

Therefore from (46) and applying Gronwall's inequality we have

$$
\mathbb{E}\left[V_{t}^{(n)}\right] \leq \frac{C_{14}}{\sqrt{\log n}}
$$

Hence we finish the proof of Theorem 2.11. 
3.5. Proof of Theorem 2.12, To prove Theorem 2.12, we introduce the following Gronwall type inequality.

Lemma 3.8 ([8, Lemma 3.2]). Let $\left(Z_{t}\right)_{t>0}$ be a non-negative continuous stochastic process and set $V_{t}:=\sup _{s<t} Z_{s}$. Assume that for some $r>0, q \geq 1, \rho \in[1, q]$ and some constants $C_{0}$ and $\xi \geq 0$,

$$
\mathbb{E}\left[V_{t}^{r}\right] \leq C_{0} \mathbb{E}\left[\left(\int_{0}^{t} V_{s} d s\right)^{r}\right]+C_{0} \mathbb{E}\left[\left(\int_{0}^{t} Z_{s}^{\rho} d s\right)^{r / q}\right]+\xi<\infty
$$

for all $t \geq 0$. Then for each $T \geq 0$ the following statements holds:

(i) If $\rho=q$, then there exists a constant $C_{1}$ depending on $C_{0}, T, q$ and $r$ such that

$$
\mathbb{E}\left[V_{T}^{r}\right] \leq C_{1} \xi
$$

(ii) If $r \geq q$ or $q+1-\rho<r<q$ hold, then there exists constant $C_{2}$ depending on $C_{0}, T, \rho, q$ and $r$, such that

$$
\mathbb{E}\left[V_{T}^{r}\right] \leq C_{2} \xi+C_{2} \int_{0}^{T} \mathbb{E}\left[Z_{s}\right] d s .
$$

Proof of Theorem 2.12, Let $p \geq 2$. First we estimate the expectation of $\sup _{0<s<t}\left|M_{s}^{\delta, \varepsilon, n}\right|^{p}$. By using (32) and the Burkholder-Davis-Gundy inequality, for any $\bar{\delta} \in(1, \infty)$ and $\varepsilon \in(0,1)$, we have

$$
\begin{aligned}
\mathbb{E}\left[\sup _{0 \leq s \leq t}\left|M_{s}^{\delta, \varepsilon, n}\right|^{p}\right] & \leq C_{1} \mathbb{E}\left[\left\langle M^{\delta, \varepsilon, n}\right\rangle_{t}^{p / 2}\right] \\
& \leq C_{2} \mathbb{E}\left[\left(\int_{0}^{t}\left|Y_{s}^{(n)}\right|^{1+2 \alpha}+\left|U_{s}^{(n)}\right|^{1+2 \alpha}+\left|s-\eta_{n}(s)\right|^{2 \beta} d s\right)^{p / 2}\right] \\
& \leq C_{3} \mathbb{E}\left[\left(\int_{0}^{t}\left|Y_{s}^{(n)}\right|^{1+2 \alpha} d s\right)^{p / 2}\right]+\frac{C_{3}}{n^{p / 4+p \alpha / 2}}+\frac{C_{3}}{n^{p \beta}} \\
& \leq C_{4} \mathbb{E}\left[\left(\int_{0}^{t}\left|Y_{s}^{(n)}\right|^{1+2 \alpha} d s\right)^{p / 2}\right]+\frac{C_{4}}{n^{p \alpha}} .
\end{aligned}
$$

Now we estimate the expectation of $\left(V_{t}^{(n)}\right)^{p}$.

Case $1(d \geq 2$ and $\alpha=1 / 2)$ : We choose $\delta=2$ and $\varepsilon=n^{-1 / 2}$. From (43), by using the inequality $\left(\sum_{i=1}^{m} a_{i}\right)^{q} \leq m^{(q-1) \vee 0} \sum_{i=1}^{m} a_{i}^{q}$ for any $m \in \mathbb{N}, a_{i} \geq 0$ and $q>0$, we have

$$
\begin{aligned}
\left(V_{t}^{(n)}\right)^{p} \leq C_{5}\{ & \left(\int_{0}^{t} V_{s}^{(n)} d s\right)^{p}+\sum_{i=1}^{d} \int_{0}^{T}\left|b^{(i)}\left(s, X_{s}^{(n)}\right)-b^{(i)}\left(s, X_{\eta_{n}(s)}^{(n)}\right)\right|^{p} d s \\
& \left.+\frac{1}{n^{p \beta}}+\sup _{0 \leq s \leq t}\left|M_{s}^{\delta, \varepsilon, n}\right|^{p}+R^{p}(1 / 2, \delta, \varepsilon, n)+S^{p}(1 / 2, \delta, \varepsilon, n)\right\} .
\end{aligned}
$$

In the same way as in (36), for any $\alpha \in(0,1 / 2]$ we have

$$
\mathbb{E}\left[R^{p}\left(\alpha, 2, n^{-1 / 2}, n\right)\right], \mathbb{E}\left[S^{p}\left(\alpha, 2, n^{-1 / 2}, n\right)\right] \leq C_{6} n^{-p \alpha} .
$$


Using Lemma 3.5 with $q=p$, (48), (49) and (50) we have

$$
\mathbb{E}\left[\left(V_{t}^{(n)}\right)^{p}\right] \leq C_{7} \mathbb{E}\left[\left(\int_{0}^{t} V_{s}^{(n)} d s\right)^{p}\right]+C_{7} \mathbb{E}\left[\left(\int_{0}^{t}\left|Y_{s}^{(n)}\right|^{2} d s\right)^{p / 2}\right]+\frac{C_{7}}{\sqrt{n}} .
$$

From Lemma 3.8(i) with $r=p, \rho=q=2$ and $\xi=C_{7} n^{-1 / 2}$, we obtain

$$
\mathbb{E}\left[\left(V_{t}^{(n)}\right)^{p}\right] \leq \frac{C_{8}}{\sqrt{n}}
$$

Case $2(d=1)$ : From (46), we have

$$
\begin{aligned}
\left(V_{t}^{(n)}\right)^{p} \leq C_{9}\left\{\left(\int_{0}^{t} V_{s}^{(n)} d s\right)^{p}\right. & +\int_{0}^{T}\left|b\left(s, X_{s}^{(n)}\right)-b\left(s, X_{\eta_{n}(s)}^{(n)}\right)\right|^{p} d s \\
& \left.+\frac{1}{n^{p \beta}}+\sup _{0 \leq s \leq t}\left|M_{s}^{\delta, \varepsilon, n}\right|^{p}+R^{p}(\alpha, \delta, \varepsilon, n)\right\} .
\end{aligned}
$$

For $\alpha=1 / 2$, we can show the statement in the same way as in Case 1 .

For $\alpha \in(0,1 / 2)$, we also take $\delta=2$ and $\varepsilon=n^{-1 / 2}$. By using (48), (50) and Lemma 3.5 with $q=p$ we have

$$
\mathbb{E}\left[\left(V_{t}^{(n)}\right)^{p}\right] \leq C_{10} \mathbb{E}\left[\left(\int_{0}^{t} V_{s}^{(n)} d s\right)^{p}\right]+C_{10} \mathbb{E}\left[\left(\int_{0}^{t}\left|Y_{s}^{(n)}\right|^{1+2 \alpha} d s\right)^{p / 2}\right]+\frac{C_{10}}{n^{p \alpha}}+\frac{C_{10}}{\sqrt{n}} .
$$

From Lemma 3.8(ii) with $r=p, q=2, \rho=1+2 \alpha$ and $\xi=C_{10} n^{-p \alpha}+C_{10} n^{-1 / 2}$, we have

$$
\mathbb{E}\left[\left(V_{t}^{(n)}\right)^{p}\right] \leq \frac{C_{11}}{n^{p \alpha}}+\frac{C_{11}}{\sqrt{n}}+C_{11} \int_{0}^{T} \mathbb{E}\left[\left|Y_{s}^{(n)}\right|\right] d s \leq C_{12}\left\{\frac{1}{n^{p \alpha}}+\frac{1}{\sqrt{n}}+\frac{1}{n^{\alpha}}\right\} \leq \frac{C_{13}}{n^{\alpha}} .
$$

For $\alpha=0$, we choose $\delta=n^{1 / 3}$ and $\varepsilon=(\log n)^{-1}$. In the same way as in (38), we have

$$
\mathbb{E}\left[R^{p}\left(0, n^{1 / 3},(\log n)^{-1}, n\right)\right] \leq \frac{C_{14}}{(\log n)^{p}} .
$$

Using Lemma 3.5 with $q=p$, (47), (50) and (51) we obtain

$$
\mathbb{E}\left[\left(V_{t}^{(n)}\right)^{p}\right] \leq C_{15} \mathbb{E}\left[\left(\int_{0}^{t} V_{s}^{(n)} d s\right)^{p}\right]+C_{15} \mathbb{E}\left[\left(\int_{0}^{t}\left|Y_{s}^{(n)}\right| d s\right)^{p / 2}\right]+\frac{C_{15}}{(\log n)^{p}} .
$$

From Lemma 3.8(ii) with $r=p, q=2, \rho=1$ and $\xi=C_{15}(\log n)^{-p}$, we have

$$
\mathbb{E}\left[\left(V_{t}^{(n)}\right)^{p}\right] \leq \frac{C_{16}}{(\log n)^{p}}+C_{16} \int_{0}^{T} \mathbb{E}\left[\left|Y_{s}^{(n)}\right|\right] d s \leq \frac{C_{17}}{\log n} .
$$

Hence the proof of the theorem is complete.

3.6. Proof of Corollaries 2.14 and 2.15. Using the same argument as in ([18, Theorem 2.1]), one can establish the Gaussian bound for the density of $\tilde{X}_{t}^{(n)}$ for $d=1$, and for the density of $\bar{X}_{t}^{(n)}$ for $d \geq 1$. Hence we can prove Corollaries 2.14 and 2.15 by using the same method as in sections 3.4 and 3.5. 
3.7. Proof of Proposition 2.2, (i) It is easy to prove that $\mathcal{A}$ is a vector space over $\mathbb{R}$.

(ii) Let $g:[0, T] \times \mathbb{R}^{d} \rightarrow \mathbb{R}$ be a bounded measurable function and let $g(t, \cdot)$ : $\mathbb{R}^{d} \rightarrow \mathbb{R}$ be monotone in each variable separately. Let $\rho(x)$ be the density function of the $d$-dimensional standard normal distribution, i.e. $\rho(x):=e^{-|x|^{2} / 2} /(2 \pi)^{d / 2}$ and let the sequence $\left(\rho_{N}\right)_{N \in \mathbb{N}}$ be defined by $\rho_{N}(x):=N^{d} \rho(N x)$. Finally, we set $g_{N}(t, x):=\int_{\mathbb{R}^{d}} g(t, y) \rho_{N}(x-y) d y$ and $\|g\|_{\infty}:=\sup _{t \in[0, T], x \in \mathbb{R}^{d}}|g(t, x)|$. We will show that $\left(g_{N}\right)$ is an $\mathcal{A}$-approximation sequence of $g$. Indeed, since $\int_{\mathbb{R}^{d}} \rho_{N}(y) d y=1$, we have $\left|g_{N}(t, x)\right| \leq\|g\|_{\infty}$. Thus $\left(g_{N}\right)$ satisfies $\mathcal{A}$ (ii). Moreover, for any $L>0$, we have

$$
\begin{aligned}
\int_{|x| \leq L}\left|g_{N}(t, x)-g(t, x)\right| d x & \leq \int_{|x| \leq L} d x \int_{\mathbb{R}^{d}} d y|g(t, y)-g(t, x)| \rho_{N}(x-y) \\
& =\int_{|x| \leq L} d x \int_{\mathbb{R}^{d}} d z|g(t, x-z)-g(t, x)| \rho_{N}(z) \\
& =\int_{\mathbb{R}^{d}} d z \int_{|x| \leq L} d x\left|g\left(t, x-\frac{z}{N}\right)-g(t, x)\right| \rho(z) .
\end{aligned}
$$

For each $z \in \mathbb{R}^{d}$, we write $z=\left(z_{1}, \ldots, z_{d}\right)^{*}, z^{(0)}=0$ and $z^{(k)}=\left(z_{1}, \ldots, z_{k}, 0, \ldots, 0\right)^{*}$ for $k=1, \ldots, d$. We have

$$
\begin{aligned}
& \int_{|x| \leq L}\left|g\left(t, x-\frac{z}{N}\right)-g(t, x)\right| d x \\
& \leq \sum_{k=1}^{d} \int_{\left|x_{1}\right| \leq L} d x_{1} \ldots \int_{\left|x_{k-1}\right| \leq L} d x_{k-1} \int_{\left|x_{k+1}\right| \leq L} d x_{k+1} \cdots \int_{\left|x_{d}\right| \leq L} d x_{d} \\
& \times \int_{\left|x_{k}\right| \leq L}\left|g\left(t, x-\frac{z^{(k)}}{N}\right)-g\left(t, x-\frac{z^{(k-1)}}{N}\right)\right| d x_{k} .
\end{aligned}
$$

Since $g(t, \cdot)$ is monotone in each variable, then

$$
\begin{aligned}
& \int_{\left|x_{k}\right| \leq L}\left|g\left(t, x-\frac{z^{(k)}}{N}\right)-g\left(t, x-\frac{z^{(k-1)}}{N}\right)\right| d x_{k} \\
& =\left|\int_{\left|x_{k}\right| \leq L}\left(g\left(t, x-\frac{z^{(k)}}{N}\right)-g\left(t, x-\frac{z^{(k-1)}}{N}\right)\right) d x_{k}\right| .
\end{aligned}
$$

By the change of variable, we have

$$
\begin{aligned}
& \left|\int_{\left|x_{k}\right| \leq L}\left(g\left(t, x-\frac{z^{(k)}}{N}\right)-g\left(t, x-\frac{z^{(k-1)}}{N}\right)\right) d x_{k}\right| \\
& =\left|\left(\int_{-L-z_{k} / N}^{-L}+\int_{L-z_{k} / N}^{L}\right) g\left(t, x-\frac{z^{(k-1)}}{N}\right) d x_{k}\right| \leq \frac{2\left|z_{k}\right|\|g\|_{\infty}}{N} .
\end{aligned}
$$

Therefore

$$
\sup _{t \in[0, T]} \int_{|x| \leq L}\left|g\left(t, x-\frac{z}{N}\right)-g(t, x)\right| d x \leq \sum_{k=1}^{d} \frac{2\left|z_{k}\right|\|g\|_{\infty} L^{d-1}}{N} .
$$


This implies that

$$
\sup _{t \in[0, T]} \int_{|x| \leq L}\left|g_{N}(t, x)-g(t, x)\right| d x \leq \int_{\mathbb{R}^{d}} \sum_{k=1}^{d} \frac{2\left|z_{k}\right|\|g\|_{\infty} L^{d-1}}{N} \rho(z) d z \rightarrow 0
$$

as $N \rightarrow \infty$. Thus $\left(g_{N}\right)_{N \in \mathbb{N}}$ satisfies $\mathcal{A}(\mathrm{i})$.

Since $g(t, \cdot)$ is a monotone function in each variable separately, so is $g_{N}(t, \cdot)$. Using the integration by parts formula, we have

$$
\begin{aligned}
& \int_{\mathbb{R}^{d}}\left|\partial_{i} g_{N}(t, x+a)\right| \frac{e^{-|x|^{2} / u}}{u^{(d-1) / 2}} d x \\
& =\left|\int_{\mathbb{R}^{d-1}} d x_{1} \ldots d x_{i-1} d x_{i+1} \ldots d x_{d} \int_{\mathbb{R}} d x_{i} \partial_{i} g_{N}(t, x+a) \frac{e^{-|x|^{2} / u}}{u^{(d-1) / 2}}\right| \\
& \leq \int_{\mathbb{R}^{d}}\left|g_{N}(t, x+a)\right| \frac{2\left|x_{i}\right|}{u} \frac{e^{-|x|^{2} / u}}{u^{(d-1) / 2}} d x \\
& \leq \int_{\mathbb{R}^{d}} \frac{2\|g\|_{\infty}|x|}{\sqrt{u}} \frac{e^{-|x|^{2} / u}}{u^{d / 2}} d x=2\|g\|_{\infty} \int_{\mathbb{R}^{d}}|y| e^{-|y|^{2}} d y,
\end{aligned}
$$

where we use the change of variable $y=x / \sqrt{u}$ in the last equation. This concludes $\left(g_{N}\right)_{N \in \mathbb{N}}$ satisfies $\mathcal{A}$ (iii).

(iii) Let $\left(g_{N}\right)$ be defined as in (ii). For each $L>0$, since $g$ is Lipschitz continuous, we have

$$
\begin{aligned}
\int_{|x| \leq L}\left|g_{N}(t, x)-g(t, x)\right| d x & \leq \int_{|x| \leq L} d x \int_{\mathbb{R}^{d}} d y|g(t, y)-g(t, x)| \rho_{N}(x-y) \\
& \leq C \int_{|x| \leq L} d x \int_{\mathbb{R}^{d}} d y|y-x| \rho_{N}(x-y) \\
& =C \int_{|x| \leq L} d x \int_{\mathbb{R}^{d}} d z \frac{|z| e^{-\frac{z^{2}}{2}}}{N} \leq \frac{C}{N} \rightarrow 0
\end{aligned}
$$

as $N \rightarrow \infty$. This implies $\left(g_{N}\right)$ satisfying $\mathcal{A}(\mathrm{i})$. It is straightforward to verify that $\left(g_{N}\right)$ satisfies $\mathcal{A}($ ii) .

To check $\mathcal{A}$ (iii), we note that from the fact $\partial_{i} \rho_{N}(x)=-N^{d+2} x_{i} \rho(N x)$ and the Lipschitz property of $g$,

$$
\begin{aligned}
\left|\partial_{i} g_{N}(t, x)\right| & =\left|\int_{\mathbb{R}^{d}} g(t, y) \partial_{i} \rho_{N}(x-y) d y\right|=\left|\int_{\mathbb{R}^{d}}\{g(t, y)-g(t, x)\} \partial_{i} \rho_{N}(x-y) d y\right| \\
& \leq \int_{\mathbb{R}^{d}} N^{d+2}|y-x|\left|y_{i}-x_{i}\right| \frac{e^{-\frac{N^{2}|y-x|^{2}}{2}}}{(2 \pi)^{d / 2}} d y .
\end{aligned}
$$

The change of variable $x=y+z / N$ implies that

$$
\int_{\mathbb{R}^{d}} N^{d+2}|y-x|\left|y_{i}-x_{i}\right| \frac{e^{-\frac{N^{2}|y-x|^{2}}{2}}}{(2 \pi)^{d / 2}} d y \leq \int_{\mathbb{R}^{d}}|z| \frac{e^{-|z|^{2} / 2}}{(2 \pi)^{d / 2}} d z=C<\infty .
$$

Hence for any $a \in \mathbb{R}^{d}$ and $u>0$,

$$
\sup _{N \in \mathbb{N}} \sum_{i=1}^{d} \int_{\mathbb{R}^{d}}\left|\partial_{i} g_{N}(t, x+a)\right| \frac{e^{-\frac{|x|^{2}}{u}}}{u^{(d-1) / 2}} d x \leq C \int_{\mathbb{R}^{d}} \frac{e^{-\frac{|x|^{2}}{u}}}{u^{(d-1) / 2}} d x \leq C \sqrt{u}
$$


holds with constant $C$ which is independent of $a$ and $u$. This concludes $\left(g_{N}\right)_{N \in \mathbb{N}}$, satisfying $\mathcal{A}($ iii).

3.8. Proof of Proposition 2.5. Let $\left(g_{N}\right)_{N \in \mathbb{N}}$ be defined as in (ii) of the previous section. Since $g \in \mathcal{L}$, there exists a positive constant $K$ such that for any $(t, x, y) \in$ $[0, T] \times \mathbb{R}^{2}$,

$$
(x-y)(g(t, x)-g(t, y)) \leq K|x-y|^{2} .
$$

Then $g_{N}$ is also a one-sided Lipschitz function. Indeed,

$$
\begin{aligned}
& (x-y)\left(g_{N}(t, x)-g_{N}(t, y)\right)=\int_{\mathbb{R}}(x-y)(g(t, x-z)-g(t, y-z)) \rho_{N}(z) d z \\
& =\int_{\mathbb{R}}\{(x-z)-(y-z)\}(g(t, x-z)-g(t, y-z)) \rho_{N}(z) d z \leq K|x-y|^{2} .
\end{aligned}
$$

Since for any $t \in[0, T]$ and $L>0$,

$$
\int_{|x| \leq L}\left|g_{N}(t, x)-g(t, x)\right| d x \leq \int_{\mathbb{R}} \rho(z) \int_{|x| \leq L}\left|g\left(t, x-\frac{z}{N}\right)-g(t, x)\right| d x d z
$$

and $\sup _{t \in[0, T]}|g(t, x-z / N)-g(t, x)| \rightarrow 0$ almost every $x$, from the dominated convergence theorem, we have

$$
\sup _{t \in[0, T]} \int_{|x| \leq L}\left|g_{N}(t, x)-g(t, x)\right| d x \rightarrow 0 .
$$

Thus $\left(g_{N}\right)_{N \in \mathbb{N}}$ satisfies $\mathcal{A}(i)$.

Let $a \in \mathbb{R}, u>0$ and $t \in[0, T]$. Since $\mathbb{R}=\bigcup_{l=-\infty}^{\infty}[l \sqrt{u},(l+1) \sqrt{u})$, we have

$$
\begin{aligned}
\int_{\mathbb{R}}\left|g_{N}^{\prime}(t, x+a)\right| e^{-\frac{x^{2}}{u}} d x & =\sum_{l=-\infty}^{\infty} \int_{l \sqrt{u}}^{(l+1) \sqrt{u}}\left|g_{N}^{\prime}(t, x+a)\right| e^{-\frac{x^{2}}{u}} d x \\
& \leq \sum_{l=-\infty}^{\infty} e^{-l^{2}} \int_{l \sqrt{u}}^{(l+1) \sqrt{u}}\left|g_{N}^{\prime}(t, x+a)\right| d x .
\end{aligned}
$$

We prove that for any $l \in \mathbb{Z}$,

$$
I_{l}(a, u):=\int_{l \sqrt{u}}^{(l+1) \sqrt{u}}\left|g_{N}^{\prime}(t, x+a)\right| d x \leq 2\left(\|g\|_{\infty}+K \sqrt{u}\right) .
$$

We fix $t \in[0, T]$ and write $[l \sqrt{u},(l+1) \sqrt{u})=\bigcup_{i \in \mathbb{N}}\left[x_{2 i}, x_{2 i+1}\right) \cup \bigcup_{j \in \mathbb{N}}\left[y_{2 j}, y_{2 j+1}\right)$ where

$g_{N}^{\prime}(t, x+a) \geq 0$ if $x+a \in\left[x_{2 i}, x_{2 i+1}\right)$ and $g_{N}^{\prime}(t, x+a)<0$ if $x+a \in\left[y_{2 j}, y_{2 j+1}\right)$.

Then we have

$$
\begin{aligned}
I_{l}(a, u) & =\sum_{i \in \mathbb{N}}\left(g_{N}\left(t, x_{2 i+1}+a\right)-g_{N}\left(t, x_{2 i}+a\right)\right) \\
& \left.+\sum_{j \in \mathbb{N}}\left(g_{N}\left(t, y_{2 j}+a\right)\right)-g_{N}\left(t, y_{2 j+1}+a\right)\right) \\
& =: I_{l}^{1}(a, u)+I_{l}^{2}(a, u) .
\end{aligned}
$$

Since $g_{N}$ is a one-sided Lipschitz function, we have

$$
\left(\left(x_{2 i+1}+a\right)-\left(x_{2 i}+a\right)\right)\left(g_{N}\left(t, x_{2 i+1}+a\right)-g_{N}\left(t, x_{2 i}+a\right)\right) \leq K\left|x_{2 i+1}-x_{2 i}\right|^{2} .
$$


Therefore, we have $\left(g_{N}\left(t, x_{2 i+1}+a\right)-g_{N}\left(t, x_{2 i}+a\right)\right) \leq K\left(x_{2 i+1}-x_{2 i}\right)$. Hence we obtain

$$
I_{l}^{1}(a, u)=\sum_{i \in \mathbb{N}}\left(g_{N}\left(t, x_{2 i+1}+a\right)-g_{N}\left(t, x_{2 i}+a\right)\right) \leq K \sqrt{u} .
$$

Finally, we estimate $I_{l}^{2}(a, u)$. Since $g_{N}$ is bounded, $\mid g_{N}(t,(l+1) \sqrt{u}+a)-g_{N}(t, l \sqrt{u}+$ $a) \mid \leq 2\|g\|_{\infty}$. Hence, we have

$$
\begin{aligned}
I_{l}^{2}(a, u) & \left.=\sum_{i \in \mathbb{N}}\left(g_{N}\left(t, x_{2 i+1}+a\right)\right)-g_{N}\left(t, x_{2 i}+a\right)\right) \\
& -\left(g_{N}(t,(l+1) \sqrt{u}+a)-g_{N}(t, l \sqrt{u}+a)\right) \\
& \leq K \sqrt{u}+2\|g\|_{\infty} .
\end{aligned}
$$

Therefore, $I_{l}(a, u) \leq 2\left(\|g\|_{\infty}+K \sqrt{u}\right)$. This concludes $\left(g_{N}\right)_{N \in \mathbb{N}}$ satisfying $\mathcal{A}($ iii).

\section{ACKNOWLEDGMENTS}

The authors are very grateful to Professor Arturo Kohatsu-Higa for suggesting this problem, and for fruitful discussions. We thank Aurélien Alfonsi and our Laboratory members for good advice. We also thank the referees for their comments which helped to improve the readability of the paper.

\section{REFERENCES}

[1] A. Foroush Bastani and M. Tahmasebi, Strong convergence of split-step backward Euler method for stochastic differential equations with non-smooth drift, J. Comput. Appl. Math. 236 (2012), no. 7, 1903-1918, DOI 10.1016/j.cam.2011.10.023. MR.2863524

[2] V. E. Beneš, L. A. Shepp, and H. S. Witsenhausen, Some solvable stochastic control problems, Stochastics 4 (1980/81), no. 1, 39-83, DOI 10.1080/17442508008833156. MR.587428 (81m:93084)

[3] K. S. Chan and O. Stramer, Weak consistency of the Euler method for numerically solving stochastic differential equations with discontinuous coefficients, Stochastic Process. Appl. 76 (1998), no. 1, 33-44, DOI 10.1016/S0304-4149(98)00020-9. MR1638015 (99f:60108)

[4] A. S. Cherny and H.-J. Engelbert, Singular Stochastic Differential Equations, Lecture Notes in Mathematics, vol. 1858, Springer-Verlag, Berlin, 2005. MR2112227 (2005j:60002)

[5] M. B. Giles, Multilevel Monte Carlo path simulation, Oper. Res. 56 (2008), no. 3, 607-617, DOI 10.1287/opre.1070.0496. MR2436856 (2009g:65008)

[6] I. Gyöngy, A note on Euler's approximations, Potential Anal. 8 (1998), no. 3, 205-216, DOI 10.1023/A:1008605221617. MR1625576 (99d:60060)

[7] I. Gyöngy and N. Krylov, Existence of strong solutions for Itô's stochastic equations via approximations, Probab. Theory Related Fields 105 (1996), no. 2, 143-158, DOI 10.1007/BF01203833. MR1392450(97h:60058)

[8] I. Gyöngy and M. Rásonyi, A note on Euler approximations for SDEs with Hölder continuous diffusion coefficients, Stochastic Process. Appl. 121 (2011), no. 10, 2189-2200, DOI 10.1016/j.spa.2011.06.008. MR2822773

[9] N. Halidias and P. E. Kloeden, A note on the Euler-Maruyama scheme for stochastic differential equations with a discontinuous monotone drift coefficient, BIT 48 (2008), no. 1, 51-59, DOI 10.1007/s10543-008-0164-1. MR2386114(2009c:60150)

[10] M. Hutzenthaler, A. Jentzen, and P. E. Kloeden, Strong and weak divergence in finite time of Euler's method for stochastic differential equations with non-globally Lipschitz continuous coefficients, Proc. R. Soc. Lond. Ser. A Math. Phys. Eng. Sci. 467 (2011), no. 2130, 15631576, DOI 10.1098/rspa.2010.0348. MR2795791 (2012g:65012)

[11] K. Itô and H. P. McKean Jr., Diffusion Processes and Their Sample Paths, Springer-Verlag, Berlin-New York, 1974. Second printing, corrected; Die Grundlehren der mathematischen Wissenschaften, Band 125. MR0345224 (49 \#9963) 
[12] I. Karatzas and S. E. Shreve, Brownian Motion and Stochastic Calculus, 2nd ed., Graduate Texts in Mathematics, vol. 113, Springer-Verlag, New York, 1991. MR1121940 (92h:60127)

[13] P. E. Kloeden and E. Platen, Numerical Solution of Stochastic Differential Equations, Applications of Mathematics (New York), vol. 23, Springer-Verlag, Berlin, 1992. MR1214374 (94b:60069)

[14] A. Kohatsu-Higa and K. Yasuda, An Ornstein-Uhlenbeck-type process which satisfies sufficient conditions for a simulation-based filtering procedure, Malliavin calculus and stochastic analysis, Springer Proc. Math. Stat., vol. 34, Springer, New York, 2013, pp. 173-193, DOI 10.1007/978-1-4614-5906-4_8. MR3070444

[15] A. Kohatsu-Higa, A. Makhlouf, and H. L. Ngo, Approximations of non-smooth integral type functionals of one dimensional diffusion processes, Stochastic Process. Appl. 124 (2014), no. 5, 1881-1909, DOI 10.1016/j.spa.2014.01.003. MR.3170228

[16] N. V. Krylov and M. Röckner, Strong solutions of stochastic equations with singular time dependent drift, Probab. Theory Related Fields 131 (2005), no. 2, 154-196, DOI 10.1007/s00440-004-0361-z. MR2117951 (2005k:60209)

[17] N. V. Krylov, On Itô stochastic differential equations, Theory of Probab. Appl., 14, No. 2, 330-336 (1969)

[18] V. Lemaire and S. Menozzi, On some non asymptotic bounds for the Euler scheme, Electron. J. Probab. 15 (2010), no. 53, 1645-1681. MR2735377 (2012b:60237)

[19] R. Mikulevičius and E. Platen, Rate of convergence of the Euler approximation for diffusion processes, Math. Nachr. 151 (1991), 233-239, DOI 10.1002/mana.19911510114. MR.1121206 (93b:60122)

[20] H. Schurz, Stability, Stationarity, and Boundedness of some Implicit Numerical Methods for Stochastic Differential Equations and Applications, Logos Verlag Berlin, Berlin, 1997. MR.1991701

[21] A. Ju. Veretennikov, Strong solutions and explicit formulas for solutions of stochastic integral equations (Russian), Mat. Sb. (N.S.) 111(153) (1980), no. 3, 434-452, 480. MR568986 (82f:60147)

[22] T. Yamada and S. Watanabe, On the uniqueness of solutions of stochastic differential equations., J. Math. Kyoto Univ. 11 (1971), 155-167. MR0278420 (43 \#4150)

[23] L. Yan, The Euler scheme with irregular coefficients, Ann. Probab. 30 (2002), no. 3, 11721194, DOI 10.1214/aop/1029867124. MR 1920104 (2003f:60115)

[24] A. K. Zvonkin, A transformation of the phase space of a diffusion process that will remove the drift (Russian), Mat. Sb. (N.S.) 93(135) (1974), 129-149, 152. MR0336813 (49 \#1586)

Department of Mathematics and Informatics, Hanoi National University of EducaTion, 136 Xuan Thuy, Cau Giay, Hanoi, Vietnam

E-mail address: ngolong@hnue.edu.vn

Department of Mathematical Sciences, Ritsumeikan University, 1-1-1 Nojihigashi, KusAtsu, Shiga, 525-8577, JAPAN

E-mail address: dai.taguchi.dai@gmail.com 